\title{
SHELLS OF MATRICES IN INDEFINITE INNER PRODUCT SPACES*
}

\author{
VLADIMIR BOLOTNIKOV ${ }^{\dagger}$, CHI-KWONG LI ${ }^{\dagger \ddagger}$, PATRICK R. MEADE ${ }^{\dagger \S}$, \\ CHRISTIAN MEHL $\uparrow$, AND LEIBA RODMAN ${ }^{\dagger} \|$
}

\begin{abstract}
The notion of the shell of a Hilbert space operator, which is a useful generalization (proposed by Wielandt) of the numerical range, is extended to operators in spaces with an indefinite inner product. For the most part, finite dimensional spaces are considered. Geometric properties of shells (convexity, boundedness, being a subset of a line, etc.) are described, as well as shells of operators in two dimensional indefinite inner product spaces. For normal operators, it is conjectured that the shell is convex and its closure is polyhedral; the conjecture is proved for indefinite inner product spaces of dimension at most three, and for finite dimensional inner product spaces with one positive eigenvalue.
\end{abstract}

Key words. Indefinite inner products, Numerical range, Shells, Normal matrices.

AMS subject classifications. 15A60, 15A63, 15A57

1. Introduction. Let $H \in \mathbb{C}^{n \times n}$ be a Hermitian matrix. Define the sesquilinear form (indefinite inner product) associated with $H$ by

$$
[x, y]_{H}=y^{*} H x, \quad x, y \in \mathbb{C}^{n},
$$

where $y^{*}$ denotes the conjugate transpose of the vector $y$. For a matrix $A \in \mathbb{C}^{n \times n}$, let

$$
W_{H}(A)=\left\{\frac{[A v, v]_{H}}{[v, v]_{H}}: v \in \mathbb{C}^{n},[v, v]_{H} \neq 0\right\} \subseteq \mathbb{C},
$$

and

$$
\mathcal{S}_{H}(A)=\left\{\left(\frac{[A v, v]_{H}}{[v, v]_{H}}, \frac{[A v, A v]_{H}}{[v, v]_{H}}\right): v \in \mathbb{C}^{n},[v, v]_{H} \neq 0\right\} \subseteq \mathbb{C} \times \mathbb{R} .
$$

When $H=I_{n}$, these reduce to the (classical) numerical range $W(A)$ and the DavisWielandt shell $\mathcal{S}(A)$ of the matrix $A$, which have been studied extensively; see $[9$, Chapter 1], [3, 4, 10, 2]. These concepts are useful in studying matrices or operators because of the interesting interplay between the algebraic properties of the matrix $A$ and the geometrical properties of the sets $W_{H}(A)$ and $\mathcal{S}_{H}(A)$. For example, the following properties are well known when $H=I_{n}$; see [9, Chapter 1].

* Received by the editors on 25 January 2002. Final manuscript accepted on 1 May 2002. Handling Editor: Ludwig Elsner.

$\dagger$ College of William and Mary, Department of Mathematics, P.O.Box 8795, Williamsburg, VA 23187-8795 (vladi@math.wm.edu, ckli@math.wm.edu, prmead@mail.wm.edu, lxrodm@math.wm.edu).

¥ The research of this author is partially supported by NSF Grant DMS 0071994.

$\S$ The research of this author was done as an undergraduate at William and Mary, and was partially supported by NSF Grant DMS 9988579.

๑ Fakultät II; Institut für Mathematik, Technische Universität Berlin, D-10623 Berlin, Germany (mehl@math.tu-berlin.de).

$\|$ The research of this author is partially supported by NSF Grant DMS-9988579. 
(1) $W\left(\alpha A+\beta I_{n}\right)=\alpha W(A)+\{\beta\}$ for any $\alpha, \beta \in \mathbb{C}$.

(2) $W\left(U^{*} A U\right)=W(A)$ for any unitary matrix $U$.

(3) $W(A)$ is convex.

(4) $W(A)=\{\lambda\}$ if and only if $A=\lambda I$.

(5) $W(A) \subseteq \mathbb{R}$ if and only if $A$ is Hermitian.

(6) $W(A)$ is a line segment if and only if $\alpha A+\beta I$ is Hermitian for some $\alpha, \beta \in \mathbb{C}$ with $\alpha \neq 0$.

(7) If $A$ is normal, then $W(A)$ is the convex hull of the eigenvalues. The converse is true if $n \leq 4$.

Many of these properties have been extended to $W_{H}(A)$ in [14]; see also [11, 12]. The purpose of this paper is to develop corresponding results for $\mathcal{S}_{H}(A)$ for a general $H$.

Since $W_{H}(A)$ is the image of $\mathcal{S}_{H}(A)$ under the projection $(z, r) \mapsto z$, one expects that $\mathcal{S}_{H}(A)$ can tell more about $A$ than $W_{H}(A)$. In fact, in the classical case, we have the following intriguing result; see [3].

(8) $\mathcal{S}(A)$ is a polyhedron, i.e., the convex hull of a finite number of points in $\mathbb{C} \times \mathbb{R}$, if and only if $A$ is normal.

Studying possible extensions of this result for general $\mathcal{S}_{H}(A)$, and additional geometric properties of $\mathcal{S}_{H}(A)$ for matrices $A$ that are normal with respect to the sesquilinear form $[\cdot, \cdot]$, is one of the main objectives of our study.

The paper is organized as follows. In Section 2, we give some preliminary definitions and notations; we also describe some general approaches for our study and some related concepts. In Section 3, we prove results relating algebraic properties of $A$ and geometrical properties of the set

$$
\mathcal{S}_{H}^{+}(A)=\left\{\left(\frac{[A v, v]_{H}}{[v, v]_{H}}, \frac{[A v, A v]_{H}}{[v, v]_{H}}\right): v \in \mathbb{C}^{n},[v, v]_{H}>0\right\},
$$

closely related to $\mathcal{S}_{H}(A)$. In Sections 4 and 5 , we study whether one can extend property (8) to the general case. It is shown by examples that $\mathcal{S}_{H}^{+}(A)$ need not be closed nor bounded, even if $A$ is assumed to be normal with respect to $[\cdot, \cdot]_{H}$, or in short $H$-normal; see (2.5) for the definition. We prove that for an $H$-normal $A$ in the following two cases: (a) $n \leq 3$; (b) $H$ is invertible with only one positive eigenvalue, the set $\mathcal{S}_{H}^{+}(A)$ is convex and its closure is either the whole of $\mathbb{C} \times \mathbb{R} \cong \mathbb{R}^{1 \times 3}$ or the intersection of finitely many closed halfspaces. We conjecture that this property is valid for all $H$-normal operators in finite dimensional indefinite inner product spaces. In Section 6, several results of previous sections are extended to operators on infinite dimensional inner product spaces. Section 7 contains a Maple program that we used in the initial stage of our project to compute examples of shells, and to formulate and check conjectures.

Throughout the paper, we denote by $H$ a fixed $n \times n$ Hermitian matrix which is not negative semidefinite. In various sections, we may assume additional hypotheses about $H$. We use $\operatorname{Conv}(S)$ to denote the convex hull of the set $S$. For a given matrix (or vector) $X, X^{T}$ and $X^{*}$ stand for the transpose and the conjugate transpose, respectively. Diagonal matrices with entries $h_{1}, \ldots, h_{n}$ on the main diagonal are written as $\operatorname{diag}\left(h_{1}, \ldots, h_{n}\right)$. A complex number $z$ is written $z=\operatorname{Re}(z)+i \operatorname{Im}(z)$, where $\operatorname{Re}(z)$ and $\operatorname{Im}(z)$ are real. Finally, we denote by $\mathbb{R}, \mathbb{R}^{+}, \mathbb{R}_{0}^{+}$, and $\mathbb{C}$ the sets of 
real numbers, positive real numbers, nonnegative real numbers, and complex numbers, respectively.

\section{Preliminaries. Let}

$$
\mathcal{S}_{H}^{-}(A)=\left\{\left([A v, v]_{H},[A v, A v]_{H}\right): v \in \mathbb{C}^{n},[v, v]_{H}=-1\right\}
$$

and

$$
\mathcal{S}_{H}^{+}(A)=\left\{\left([A v, v]_{H},[A v, A v]_{H}\right): v \in \mathbb{C}^{n},[v, v]_{H}=1\right\}
$$

Then

$$
\mathcal{S}_{H}(A)=\mathcal{S}_{H}^{-}(A) \cup \mathcal{S}_{H}^{+}(A)
$$

We always assume that the set of vectors $v$ such that $[v, v]_{H}=1$ (resp., $[v, v]_{H}=-1$ ) is not empty when considering $\mathcal{S}_{H}^{+}(A)$ (resp., $\mathcal{S}_{H}^{-}(A)$ ). All the sets $\mathcal{S}_{H}(A), \mathcal{S}_{H}^{+}(A)$ and $\mathcal{S}_{H}^{-}(A)$ will be referred to as the Davis-Wielandt shells of $A$ with respect to $H$ in our study. Since $\mathcal{S}_{H}^{-}(A)=\mathcal{S}_{-H}^{+}(A)$, we focus only on $\mathcal{S}_{H}^{+}(A)$. We will often identify $\mathbb{C} \times \mathbb{R}$ with $\mathbb{R}^{1 \times 3}$ via $(a+i b, c) \mapsto(a, b, c)$ in $(1.2)$, and in analogous formulas for $\mathcal{S}_{H}^{-}(A)$ and $\mathcal{S}_{H}^{+}(A)$. Also, it will be convenient to represent $\mathcal{S}_{H}^{+}(A)$ in matrix notation:

$\mathcal{S}_{H}^{+}(A)=\left\{\left(x^{*}\left(\frac{H A+A^{*} H}{2}\right) x, x^{*}\left(\frac{H A-A^{*} H}{2 i}\right) x, x^{*} A^{*} H A x\right): x \in \mathbb{C}^{n}, x^{*} H x=1\right\}$.

If $H$ is invertible, the $H$-adjoint of $A$ is denoted by $A^{[*]}$, which is uniquely defined by the relation

$$
[A u, v]=\left[u, A^{[*]} v\right] \quad u, v \in \mathbb{C}^{n}
$$

and it can be expressed explicitly in terms of $A$ and $H$ by

$$
A^{[*]}:=H^{-1} A^{*} H \text {. }
$$

A matrix $A$ is called $H$-selfadjoint, $H$-unitary, or $H$-normal if

$$
A=A^{[*]}, \quad A^{[*]} A=I_{n} \quad \text { or } \quad A A^{[*]}=A^{[*]} A,
$$

respectively. In view of (2.2), the three equalities (2.3) are equivalent, respectively, to

$$
H A=A^{*} H, \quad A^{*} H A=H \quad \text { or } \quad A^{*} H A=H A H^{-1} A^{*} H .
$$

In the case when $\operatorname{det} H=0$, relation (2.1) does not determine a unique $H$-adjoint of $A$ and of course formula (2.2) does not make sense anymore. However, one can introduce $H$-selfadjoint and $H$-unitary matrices using the first two relations in (2.4).

The notion of $H$-normality introduced in [14] is a little bit more delicate. A matrix $A$ is called $H$-normal if

$$
A^{*} H A=H A H^{[-1]} A^{*} H,
$$


where $H^{[-1]}$ denotes the Moore-Penrose pseudoinverse of $H$, which is uniquely defined by the conditions

$$
H^{[-1]} H H^{[-1]}=H^{[-1]}, \quad H H^{[-1]} H=H, \quad H^{[-1]} H=H H^{[-1]}=\mathbf{P}_{\text {Range } H},
$$

and where $\mathbf{P}_{\text {Range } H}$ stands for the orthogonal projection onto Range $H$.

We start with some useful facts:

Proposition 2.1. Let $A \in \mathbb{C}^{n \times n}$.

(a) If $r>0$ and $t \in \mathbb{R}$, then

$$
\mathcal{S}_{H}^{+}\left(r e^{i t} A\right)=\mathcal{S}_{H}^{+}(A)\left[\begin{array}{ccc}
r \cos t & r \sin t & 0 \\
-r \sin t & r \cos t & 0 \\
0 & 0 & r^{2}
\end{array}\right] .
$$

(b) If $\lambda \in \mathbb{C}$, then

$$
\mathcal{S}_{H}^{+}(A+\lambda I)=\left(\operatorname{Re}(\lambda), \operatorname{Im}(\lambda),|\lambda|^{2}\right)+\mathcal{S}_{H}^{+}(A)\left[\begin{array}{ccc}
1 & 0 & \bar{\lambda}+\lambda \\
0 & 1 & i(\bar{\lambda}-\lambda) \\
0 & 0 & 1
\end{array}\right] .
$$

(c) Let $T$ be any invertible $n \times n$ matrix. Then

$$
\mathcal{S}_{H}^{+}(A)=\mathcal{S}_{T^{*} H T}^{+}\left(T^{-1} A T\right) .
$$

Proof. To check (c), just replace $T x$ by $y$ in the following chain of equalities:

$$
\begin{aligned}
\mathcal{S}_{T^{*} H T}^{+}\left(T^{-1} A T\right) & =\left\{\left(x^{*} T^{*} H A T x, x^{*} T^{*} A^{*} H A T x\right): x^{*} T^{*} H T x=1\right\} \\
& =\left\{\left(y^{*} H A y, y^{*} A^{*} H A y\right): y^{*} H y=1\right\}=\mathcal{S}_{H}^{+}(A) .
\end{aligned}
$$

Statements (a) and (b) follow from the definitions of $\mathcal{S}_{H}^{+}\left(r e^{i t} A\right)$ and $\mathcal{S}_{H}^{+}(A+\lambda I)$ upon simple algebraic manipulations.

Thus, the transformation

$$
(A, H) \mapsto\left(T^{-1} A T, T^{*} H T\right)
$$

preserves shells. This transformation also preserves the classes of $H$-selfadjoint, $H-$ unitary, and $H$-normal matrices:

Lemma 2.2. Let $H \in \mathbb{C}^{n \times n}$ be Hermitian and possibly singular and let $T \in \mathbb{C}^{n \times n}$ be invertible. Then $A \in \mathbb{C}^{n \times n}$ is $H$-normal (resp., $H$-selfadjoint, or $H$-unitary) if and only if $\widehat{A}$ is $\widehat{H}$-normal (resp., $\widehat{H}$-selfadjoint, or $\widehat{H}$-unitary), where $\widehat{A}=T^{-1} A T$ and $\widehat{H}=T^{*} H T$.

Proof. We verify the lemma only for the $H$-normal matrices. First we note that the Moore-Penrose pseudoinverse of $\widehat{H}$ equals $\widehat{H}^{[-1]}=T^{-1} H^{[-1]}\left(T^{-1}\right)^{*}$. Therefore, on account of (2.5),

$$
\begin{aligned}
\widehat{H} \widehat{A} \widehat{H}^{[-1]} \widehat{A}^{*} \widehat{H} & =T^{*} H T T^{-1} A T T^{-1} H^{[-1]}\left(T^{-1}\right)^{*} T^{*} A^{*}\left(T^{-1}\right)^{*} T^{*} H T \\
& =T^{*} H A H^{[-1]} A^{*} H T=T^{*} A^{*} H A T=\widehat{A}^{*} \widehat{H} \widehat{A}
\end{aligned}
$$


which completes the proof.

THEOREM 2.3. Assume that the positive semidefinite matrix $H$ is singular, and denote by $P$ the orthogonal projection along the kernel of $H$. If $A$ is $H$-normal, then $P A P$ (considered as a linear transformation on $\left.(\operatorname{Ker} H)^{\perp}\right)$ is PHP-normal, where PHP stands for the restriction of $H$ to $(\operatorname{Ker} H)^{\perp}$, and

$$
\mathcal{S}_{H}^{+}(A)=\mathcal{S}_{P H P}^{+}(P A P) .
$$

Proof. Applying transformation (2.7), we can assume that

$$
A=\left[\begin{array}{ll}
A_{11} & A_{12} \\
A_{21} & A_{22}
\end{array}\right] \quad \text { and } \quad H=\left[\begin{array}{cc}
H_{1} & 0 \\
0 & 0
\end{array}\right],
$$

where $H_{1}$ is a positive definite matrix. Then $P A P=A_{11}, P H P=H_{1}$, and $H^{[-1]}=$ $\left[\begin{array}{cc}H_{1}^{-1} & 0 \\ 0 & 0\end{array}\right]$. Condition (2.5) now gives $A_{12}=0$ and

$$
A_{11}^{*} H_{1} A_{11}=H_{1} A_{11} H_{1}^{-1} A_{11}^{*} H_{1},
$$

which means that $P A P$ is $P H P$-normal. Finally,

$$
H A=\left[\begin{array}{cc}
H_{1} A_{11} & 0 \\
0 & 0
\end{array}\right], \quad A^{*} H A=\left[\begin{array}{cc}
A_{11}^{*} H_{1} A_{11} & 0 \\
0 & 0
\end{array}\right],
$$

and the result follows.

For Hermitian matrices $A_{1}, \ldots, A_{p} \in \mathbb{C}^{n \times n}$, we denote by $W\left(A_{1}, \ldots, A_{p}\right)$ their joint numerical range:

$$
W\left(A_{1}, \ldots, A_{p}\right)=\left\{\left(x^{*} A_{1} x, \ldots, x^{*} A_{p} x\right) \in \mathbb{R}^{p}: x \in \mathbb{C}^{n}, x^{*} x=1\right\}
$$

and by $\sigma\left(A_{1}, \ldots, A_{p}\right)$ their joint spectrum:

$$
\sigma\left(A_{1}, \ldots, A_{p}\right)=\left\{\left(a_{1}, \ldots, a_{p}\right) \in \mathbb{R}^{p}: A_{1} x=a_{1} x, \ldots, A_{p} x=a_{p} x \text { for some } x \neq 0\right\} .
$$

The next proposition, which is easy to verify, shows the connection between $\mathcal{S}_{H}^{+}(A)$ and the joint numerical range, whose study can be reduced to the $2 \times 2$ case by compression.

Proposition 2.4. Let $H A=F+i G$, where $F$ and $G$ are Hermitian, and $K=A^{*} H A$. Then

$$
\mathcal{S}_{H}^{+}(A)=\left\{(a+i b, c): a, b, c \in \mathbb{R},(a, b, c, 1) \in\left[\bigcup_{t>0} t W(F, G, K, H)\right]\right\},
$$

and

$$
W(F, G, K, H)=\bigcup_{X \in \mathbb{C}^{n \times 2}, X^{*} X=I_{2}} W\left(X^{*} F X, X^{*} G X, X^{*} K X, X^{*} H X\right) .
$$


Since the $2 \times 2$ case may be used to study the general case, it is useful to have a complete description of this low dimensional case. Recall that a subset $S$ of a real linear space has affine dimension $m$ if the set $S-v_{0}$ spans an $m-1$ dimensional subspace for some (any) $v_{0} \in S$.

Proposition 2.5. Let $A \in \mathbb{C}^{2 \times 2}$ and let $m$ be the dimension of the real subspace spanned by the set

$$
\left\{H, H A+A^{*} H, i\left(H A-A^{*} H\right), A^{*} H A\right\} .
$$

Then $\mathcal{S}_{H}^{+}(A)$, regarded as a subset of $\mathbb{R}^{1 \times 3}$, has affine dimension $m-1$. In particular, if $m=1$, then $\mathcal{S}_{H}^{+}(A)$ is a singleton. If $m>1$, then one of the following conditions holds.

(a) $H$ is positive definite; $m=2$ and $\mathcal{S}_{H}^{+}(A)$ is a nondegenerate closed line segment, equivalently, $A$ is $H$-normal and not a (complex) scalar multiple of $I_{2}$; $m=4$ and $\mathcal{S}_{H}^{+}(A)$ is an ellipsoid without interior in all other cases.

(b) $H \neq 0$ is positive semidefinite and singular; $m=4$ and $\mathcal{S}_{H}^{+}(A)$ is a paraboloid without interior.

(c) $H$ is indefinite; $m=2$ and $\mathcal{S}_{H}^{+}(A)$ is a (closed or open) half line or a whole line; $m=3$ and $\mathcal{S}_{H}^{+}(A)$ is a one-component hyperbola with interior, an open two-dimensional half plane, or a two-dimensional plane; $m=4$ and $\mathcal{S}_{H}^{+}(A)$ is a one-component hyperboloid without interior.

Proof. The proposition follows from [13, Theorem 2.1], which describes the joint numerical ranges of a $p$-tuple of $2 \times 2$ Hermitian matrices with respect to a sesquilinear form in $\mathbb{C}^{2}$. We need only to make the following observations that take into account the special form of the matrices $H A+A^{*} H, i\left(H A-A^{*} H\right)$, and $A^{*} H A$.

(a) By Proposition 2.1, we may assume $H=I_{2}$. It is easy to see that $m=1$ if $A=\lambda I, \lambda \in \mathbb{C}, m=2$ if $A \neq \lambda I$ and is normal, and $m=4$ in all other cases.

(b) Assume that $H=\left[\begin{array}{ll}1 & 0 \\ 0 & 0\end{array}\right]$, and write $A=\left[\begin{array}{ll}a & b \\ c & d\end{array}\right]$. One easily verifies that $m=1$ if $b=0$, and $m=4$ in all other cases.

(c) We assume that $H=\left[\begin{array}{rr}1 & 0 \\ 0 & -1\end{array}\right]$, and write $A=\left[\begin{array}{ll}a & b \\ c & d\end{array}\right]$. To compute the dimension $m$ of the span of the set in (2.8), we may assume that $a=-d \geq 0$; otherwise, we may replace $A$ by a matrix of the form $\mu(A-\eta I)$ for some suitable $\mu, \eta \in \mathbb{C}$ with $|\mu|=1$. We may further assume that $b \geq 0$; otherwise replace $(H, A)$ by $\left(D^{*} H D, D^{*} A D\right)=\left(H, D^{*} A D\right)$ for a suitable diagonal unitary matrix $D$. Assume first that $a=0$. Then the four matrices (2.8) take the form

$$
\left[\begin{array}{cc}
1 & 0 \\
0 & -1
\end{array}\right],\left[\begin{array}{cc}
0 & b-\bar{c} \\
b-c & 0
\end{array}\right],\left[\begin{array}{cc}
0 & i(b+\bar{c}) \\
i(-b-c) & 0
\end{array}\right],\left[\begin{array}{cc}
-|c|^{2} & 0 \\
0 & b^{2}
\end{array}\right] .
$$

It is easy to see, using the fact that the complex numbers $b-\bar{c}$ and $i(b+\bar{c})$ are linearly independent over $\mathbb{R}$ if and only if $b^{2} \neq|c|^{2}$, that $m=2$ if $b^{2}=|c|^{2}$ and $m=4$ if $b^{2} \neq|c|^{2}$. Assume now that $a \neq 0$. Then (2.8) takes the form

$$
\left[\begin{array}{cc}
1 & 0 \\
0 & -1
\end{array}\right],\left[\begin{array}{cc}
2 a & b-\bar{c} \\
b-c & 2 a
\end{array}\right],\left[\begin{array}{cc}
0 & i(b+\bar{c}) \\
i(-b-c) & 0
\end{array}\right],\left[\begin{array}{cc}
a^{2}-|c|^{2} & a(b+\bar{c}) \\
a(b+c) & b^{2}-a^{2}
\end{array}\right]
$$


If $b+\bar{c}=0$, then clearly $m=2$. If $b+\bar{c} \neq 0$, then

$$
\begin{gathered}
{\left[\begin{array}{cc}
a^{2}-|c|^{2} & a(b+\bar{c}) \\
a(b+c) & b^{2}-a^{2}
\end{array}\right]+\left(-a^{2}+s\right)\left[\begin{array}{cc}
1 & 0 \\
0 & -1
\end{array}\right]+r\left[\begin{array}{cc}
2 a & b-\bar{c} \\
b-c & 2 a
\end{array}\right]} \\
=\left[\begin{array}{cc}
0 & a(b+\bar{c})+r(b-\bar{c}) \\
a(b+c)+r(b-c) & 0
\end{array}\right],
\end{gathered}
$$

where $r=\left(-b^{2}+|c|^{2}\right) /(4 a), s=\left(|c|^{2}+b^{2}\right) / 2$. If the complex numbers

$$
a(b+\bar{c})+r(b-\bar{c}) \quad \text { and } \quad i(b+\bar{c})
$$

are linearly independent over $\mathbb{R}$, then $m=4$; otherwise, $m=3$. A simple calculation shows that the complex numbers in (2.9) are linearly independent over $\mathbb{R}$ if and only if $2 a \neq \pm\left(b^{2}-|c|^{2}\right) /|b+c|$. $\mathrm{c}$

3. Geometric properties of shells. In this section we study geometric properties of shells: convexity, degeneracy (being a singleton, or a subset of a line), boundedness.

We start with the problem of convexity. For the $2 \times 2$ case, this can be easily sorted out using the general description of shells of $2 \times 2$ matrices in Proposition 2.5.

Proposition 3.1. Let $A \in \mathbb{C}^{2 \times 2}$. Then $\mathcal{S}_{H}^{+}(A)$ is convex if and only if the matrices $H,\left(H A+A^{*} H\right), i\left(H A-A^{*} H\right)$, and $A^{*} H A$ are linearly dependent.

In general, the shell is not convex, for matrices of size larger than 2 :

EXAMPLE 3.2. Let

$$
H=\left[\begin{array}{rrr}
1 & 0 & 0 \\
0 & -1 & 0 \\
0 & 0 & 1
\end{array}\right], \quad A=\left[\begin{array}{lll}
0 & 0 & 0 \\
2 & 0 & 0 \\
0 & 0 & 0
\end{array}\right]
$$

Then $H A=F+i G$, where

$$
F=\left[\begin{array}{lll}
0 & 1 & 0 \\
1 & 0 & 0 \\
0 & 0 & 0
\end{array}\right], \quad G=\left[\begin{array}{rrr}
0 & i & 0 \\
-i & 0 & 0 \\
0 & 0 & 0
\end{array}\right], \quad \text { and } \quad A^{*} H A=\left[\begin{array}{rrr}
-4 & 0 & 0 \\
0 & 0 & 0 \\
0 & 0 & 0
\end{array}\right]
$$

Setting $x=\left[\begin{array}{lll}x_{1} & x_{2} & x_{3}\end{array}\right]^{T}$, we note that $x^{*} H x=1$ if and only if $\left|x_{1}\right|^{2}-\left|x_{2}\right|^{2}+\left|x_{3}\right|^{2}=1$. A simple calculation shows that

$$
\mathcal{S}_{H}^{+}(A)=\left\{\left(2 \operatorname{Re}\left(\overline{x_{1}} x_{2}\right), 2 \operatorname{Im}\left(\overline{x_{1}} x_{2}\right),-4\left|x_{1}\right|^{2}\right):\left|x_{1}\right|^{2}-\left|x_{2}\right|^{2}+\left|x_{3}\right|^{2}=1\right\} .
$$

This set is not convex, for instance if we take the cross-section where $-4\left|x_{1}\right|^{2}=-16$. In this case $\left|x_{2}\right|^{2}=\left|x_{1}\right|^{2}+\left|x_{3}\right|^{2}-1 \geq 3$, and therefore the cross section of $\mathcal{S}_{H}^{+}(A)$ is

$$
\left\{\left(2 \operatorname{Re}\left(\overline{x_{1}} x_{2}\right), 2 \operatorname{Im}\left(\overline{x_{1}} x_{2}\right),-16\right):\left|x_{2}\right|^{2} \geq 3\right\}=\left\{(q, r,-16): q^{2}+r^{2} \geq 48\right\},
$$

which is not convex. 
Sufficient conditions for convexity are given in the following theorem.

Theorem 3.3. Let $H A=F+i G$ be such that $F, G$ are Hermitian. Then $\mathcal{S}_{H}^{+}(A)$ is convex if any one of the following two conditions holds.

(a) The four matrices $H, F, G, A^{*} H A$ are linearly dependent.

(b) $n \geq 3$, and the span of $\left\{H, F, G, A^{*} H A\right\}$ contains a positive definite matrix.

Proof. The result follows from [13, Theorem 3.2 (a) and (b)].

We say that a set in $\mathbb{R}^{k}$ is polyhedral if it is the intersection of finitely many closed half spaces; $\mathbb{R}^{k}$ itself will also be considered a polyhedral set. Clearly, every polyhedron is a polyhedral set; a polyhedral set is a polyhedron if and only if it is bounded. Also, every polyhedral set is convex.

TheOREM 3.4. Let $A \in \mathbb{C}^{n \times n}, K=A^{*} H A$, and $H A=F+i G$ be such that $F$ and $G$ are Hermitian. Suppose there exists an invertible $T$ such that $T^{*} H T=$ $\operatorname{diag}\left(h_{1}, \ldots, h_{n}\right), T^{*} F T=\operatorname{diag}\left(f_{1}, \ldots, f_{n}\right)$ and $T^{*} G T=\operatorname{diag}\left(g_{1}, \ldots, g_{n}\right)$. Then

$$
W(F, G, K, H)=\operatorname{Conv}\left\{\left(f_{j}, g_{j}, h_{j}\left(f_{j}^{2}+g_{j}^{2}\right), h_{j}\right): 1 \leq j \leq n\right\}
$$

is a polyhedron, and thus

$$
\mathcal{S}_{H}^{+}(A)=\left\{(a+i b, c): a, b, c \in \mathbb{R},(a, b, c, 1) \in\left[\bigcup_{t>0} t W(F, G, K, H)\right]\right\}
$$

is the intersection of an affine space with a convex polyhedral cone, which is a polyhedral set.

Proof. The assertion on $W(F, G, K, H)$ is well known; see [1]. The assertion on $\mathcal{S}_{H}^{+}(A)$ follows readily from Proposition 2.4.

Matrices with degenerate shells are characterized as follows.

Theorem 3.5. Let $A \in \mathbb{C}^{n \times n}$. Then:

(a) $\mathcal{S}_{H}^{+}(A)$ is a singleton if and only if the matrix $H A$ is a (complex) scalar multiple of $H$.

(b) $\mathcal{S}_{H}^{+}(A)$ is a subset of a line if and only if $H A=u H+v G$ and $A^{*} H A=r H+s G$ for some $u, v \in \mathbb{C}, r, s \in \mathbb{R}$, and some Hermitian $G$ which is not a scalar multiple of $H$; equivalently, the matrices $H, H A+A^{*} H, i\left(H A-A^{*} H\right)$, $A^{*} H A$ span a two dimensional real subspace.

Proof. The result follows from [13, Theorem 3.5].

For boundedness of shells, we have the following result.

THEOREM 3.6. The shell $\mathcal{S}_{H}^{+}(A)$ is bounded if and only if the kernel of $H$ is $A$ invariant (this condition is obviously satisfied if $H$ is invertible) and EITHER $H$ is positive semidefinite $O R H$ is indefinite and there exists $\alpha \in \mathbb{C}$ such that $H A=\alpha H$.

Proof. The "if" part. Applying transformation (2.7), we can assume that

$$
A=\left[\begin{array}{cc}
A_{11} & 0 \\
A_{21} & A_{22}
\end{array}\right], \quad H=\left[\begin{array}{cc}
H_{1} & 0 \\
0 & 0
\end{array}\right],
$$

where $H_{1}$ is invertible. Then

$$
\mathcal{S}_{H}^{+}(A)=\mathcal{S}_{H_{1}}^{+}\left(A_{11}\right),
$$


which is clearly bounded if $H_{1}$ is positive definite, or if $H_{1} A_{11}=\alpha H_{1}$ for some $\alpha \in \mathbb{C}$.

The "only if" part. If $\mathcal{S}_{H}^{+}(A)$ is bounded, then so is the set

$$
W_{H}^{+}(A)=\left\{[A v, v]_{H}: v \in \mathbb{C}^{n},[v, v]_{H}=1\right\} .
$$

The boundedness of $W_{H}^{+}(A)$ is characterized in [11, Theorem 2.3], implying the result.

4. Normality and polyhedral shells: Semidefinite case. The purpose of this section is to study whether Property (8) in Section 1 can be extended to the general case when $H$ is positive semidefinite. First, consider the case when $H$ is positive definite. Without loss of generality, one can assume that $H=I_{n}$. We fix $A \in \mathbb{C}^{n \times n}$ along with its representation $A=F+i G$, where $F$ and $G$ are Hermitian matrices. Furthermore, $S_{H}^{+}(A)$ and $\mathcal{S}_{H}(A)$ are the same as $W\left(F, G, A^{*} A\right)$.

LEMmA 4.1. If $a+i b, a, b \in \mathbb{R}$, is an eigenvalue of $A$, then $\left(a, b, a^{2}+b^{2}\right) \in$ $W\left(F, G, A^{*} A\right)$, and the inequality $a^{2}+b^{2} \leq c$ holds for every $(a, b, c) \in W\left(F, G, A^{*} A\right)$.

Proof. Let $U$ be a unitary matrix, such that $U^{*} A U$ is of the upper triangular form with $a+i b$ lying in the $(1,1)$ position and let $u$ be the first column of $U$. Then $\left(a, b, a^{2}+b^{2}\right)=\left(u^{*} F u, u^{*} G u, u^{*} A^{*} A u\right) \in W\left(F, G, A^{*} A\right)$.

Now, suppose $v \in \mathbb{C}^{n}$ is a unit vector such that $v^{*} A v=a+i b$, then $A v=$ $(a+i b) v+d w$ for some $d \geq 0$ and some unit vector $w \in \mathbb{C}^{n}$ satisfying $v^{*} w=0$. It follows that $v^{*} A^{*} A v=a^{2}+b^{2}+d^{2} \geq a^{2}+b^{2}$.

The following two results have been established in [1].

LEMma 4.2. If $\left(a_{1}, a_{2}, a_{3}\right)$ is a vertex point of $W\left(A_{1}, A_{2}, A_{3}\right)$, then $\left(a_{1}, a_{2}, a_{3}\right) \in$ $\sigma\left(A_{1}, A_{2}, A_{3}\right)$.

Corollary 4.3. If $W\left(A_{1}, A_{2}, A_{3}\right)$ is a polyhedron, then

$$
W\left(A_{1}, A_{2}, A_{3}\right)=\operatorname{Conv}\left(\sigma\left(A_{1}, A_{2}, A_{3}\right)\right) .
$$

The next theorem was essentially proved in $[3,4]$ using a different approach.

THEOREM 4.4. Let $A=F+i G$. The following are equivalent:

(a) $W\left(F, G, A^{*} A\right)$ is a polyhedron in $\mathbb{R}^{1 \times 3}$.

(b) $W\left(F, G, A^{*} A\right)=\operatorname{Conv}\left(\sigma\left(F, G, A^{*} A\right)\right)$.

(c) $A$ is normal.

Proof. Implication (c) $\Rightarrow$ (a) follows from Theorem 3.4. Implication (a) $\Rightarrow$ (b) follows readily from Corollary 4.3. To prove implication $(b) \Rightarrow(c)$, suppose $W\left(F, G, A^{*} A\right)=\operatorname{Conv}\left(\sigma\left(F, G, A^{*} A\right)\right)$. Then $W\left(F, G, A^{*} A\right)$ is a polyhedron. We claim that every eigenvalue of $A$ is reducing. Suppose $S=\left\{a_{j}+i b_{j}: 1 \leq j \leq k\right\}$ are the distinct eigenvalues of $A$. By Lemma 4.1, we have

$$
\operatorname{Conv}\left\{\left(a_{j}, b_{j}, a_{j}^{2}+b_{j}^{2}\right): 1 \leq j \leq k\right\} \subseteq W\left(F, G, A^{*} A\right) \subseteq \operatorname{Conv}\left(\sigma\left(F, G, A^{*} A\right)\right) .
$$

Hence $\operatorname{Conv}\left\{\left(a_{j}, b_{j}, a_{j}^{2}+b_{j}^{2}\right): 1 \leq j \leq k\right\}=\operatorname{Conv}\left(\sigma\left(F, G, A^{*} A\right)\right)$. Since the function $f(x, y)=x^{2}+y^{2}$ is strictly convex on $\mathbb{R}^{1 \times 2}$, and all the points $\left(a_{j}, b_{j}, a_{j}^{2}+b_{j}^{2}\right)$ lie on its 
graph, we see that each point $\left(a_{j}, b_{j}, a_{j}^{2}+b_{j}^{2}\right)$ is a vertex of the set $\operatorname{Conv}\left\{\left(a_{j}, b_{j}, a_{j}^{2}+b_{j}^{2}\right)\right.$ : $1 \leq j \leq k\}$. By Lemma 4.2, we see that each $a_{j}+i b_{j}$ is a reducing eigenvalue of $A$. Hence $A$ is normal.

If $H$ is only positive semidefinite, we have the following result, extending Davis' theorem (statement (8) of Section 1).

TheOrem 4.5. Let $H \in \mathbb{C}^{n \times n}$ be positive semidefinite and singular. Then the following statements are equivalent for $A \in \mathbb{C}^{n \times n}$ :

(a) $\mathcal{S}_{H}^{+}(A)$ is a polyhedron.

(b) $\mathcal{S}_{H}^{+}(A)$ is a polyhedral set.

(c) $A$ is $H$-normal.

Proof. (c) $\Rightarrow$ (a) follows from Theorem 2.3 and the property (8) of Section 1. (a) $\Rightarrow(\mathrm{b})$ is trivial.

(b) $\Rightarrow$ (c). Without loss of generality, we let

$$
H=\left[\begin{array}{cc}
I_{k} & 0 \\
0 & 0
\end{array}\right], \quad A=\left[\begin{array}{ll}
A_{11} & A_{12} \\
A_{21} & A_{22}
\end{array}\right]
$$

where $A_{11}$ and $A_{22}$ are $k \times k$ and $(n-k) \times(n-k)$, respectively. Arguing by contradiction, assume that $A$ is not $H$-normal. If $A_{12}=0$, then $A_{11}$ is not $I_{k}$-normal. On the other hand, $S_{I_{k}}^{+}\left(A_{11}\right)=\mathcal{S}_{H}^{+}(A)$ (cf. the proof of Theorem 2.3), and therefore $S_{I_{k}}^{+}\left(A_{11}\right)$, being a bounded polyhedral set, is a polyhedron, a contradiction with Theorem 4.4.

Now suppose $A_{12} \neq 0$. Let $t>5\left\|A_{11}\right\|$, consider the level set

$$
S_{t}=\left\{v^{*}(H A) v: v^{*} H v=1, v^{*}\left(A^{*} H A\right) v=t^{2}\right\},
$$

which coincides with the projection on the first two components of the set

$$
S_{H}^{+}(A) \cap\left\{\left(x_{1}, x_{2}, t^{2}\right) \in \mathbb{R}^{1 \times 3}: x_{1}, x_{2} \in \mathbb{R}\right\} .
$$

Since $A_{12} \neq 0$, there exists $w \in \mathbb{C}^{n-k}$ such that $A_{12} w \neq 0$. Let $x$ be a unit vector in $\mathbb{C}^{k}$. Then there exists $\nu \in \mathbb{C}$ such that $\left\|A_{11} x+A_{12}(\nu w)\right\|=t$. If $v=\left[\begin{array}{c}x \\ \nu w\end{array}\right] \in \mathbb{C}^{n}$, then $v^{*}(H A) v \in \mathcal{S}_{t}$. Thus, $\mathcal{S}_{t} \neq \emptyset$. Clearly, $S_{t}$ is a polyhedral set. Using the Cauchy-Schwartz inequality

$$
\left|v^{*} H A v\right|=\left|\left(v^{*} H\right)(H A v)\right| \leq \sqrt{v^{*} H v} \sqrt{v^{*} A^{*} H H A v}=\sqrt{v^{*} H v} \sqrt{v^{*} A^{*} H A v}=t
$$

for every $v \in \mathbb{C}^{n}$ satisfying $v^{*} H A v \in S_{t}$, we see that the set $S_{t}$ is bounded, and therefore is a convex polygon (with interior).

Let $\mu=v^{*}(H A) v$ be a corner point of $S_{t}$. Writing $v=\left[\begin{array}{l}x \\ y\end{array}\right]$, where $x \in \mathbb{C}^{k}$, $y \in \mathbb{C}^{n-k}$, we have

$\mu=x^{*} A_{11} x+x^{*} A_{12} y, \quad v^{*} H v=x^{*} x=1, \quad$ and $\quad\left\|A_{11} x+A_{12} y\right\|^{2}=v^{*} A^{*} H A v=t^{2}$. 
We claim that $x^{*} A_{12} y=0$. Suppose not, i.e., let $x^{*} A_{12} y \neq 0$. We construct a real positive function $\delta(\theta)$, where $\theta \in(-r, r)$ for some $r>0$, such that $\delta(0)=1$, and

$$
\left\|A_{11} x+A_{12}\left(e^{i \theta} \delta(\theta) y\right)\right\|=t, \quad-r<\theta<r,
$$

as follows. Letting $x^{*} A_{11}^{*} A_{12} y=u+i w, u, w \in \mathbb{R}$, a calculation shows that $\delta(\theta)$ satisfies the quadratic equation

$$
\left((\delta(\theta))^{2}-1\right) y^{*} A_{12}^{*} A_{12} y+2 \delta(\theta)((\cos \theta) u-(\sin \theta) w)-2 u=0 .
$$

Note that $A_{12} y \neq 0$, because $x^{*} A_{12} y \neq 0$. Since $\left\|A_{11} x+A_{12} y\right\|=t>5\left\|A_{11}\right\|$, we see that the product of the roots of the above equation is

$-1-2 u /\left\|A_{12} y\right\|^{2} \leq-1+2\left\|A_{11} x\right\|\left\|A_{12} y\right\| /\left\|A_{12} y\right\|^{2} \leq-1+2\left\|A_{11}\right\| /\left(t-\left\|A_{11}\right\|\right)<0$.

Thus, equation (4.2) always has a positive root $\delta(\theta)$ and a negative root. Therefore, $\delta(\theta)$ is a differentiable (even analytic) function of $\theta$. Consider the curve

$$
\mu(\theta)=x^{*} A_{11} x+e^{i \theta} \delta(\theta) x^{*} A_{12} y, \quad-r<\theta<r .
$$

By $(4.1), \mu(\theta) \in S_{t}$; also $\mu(0)=\mu$. Since

$$
\lim _{\theta \rightarrow 0}(\mu(\theta)-\mu) / \theta=\left(i+\delta^{\prime}(0)\right) x^{*} A_{12} y
$$

the line $\left\{\mu+s\left(i+\delta^{\prime}(0)\right) x^{*} A_{12} y: s \in \mathbb{R}\right\}$ is the tangent to the curve $\mu(\theta)$ at $\mu$, a contradiction with $\mu$ being a corner of $S_{t}$. Thus, $x^{*} A_{12} y=0$, and $\mu$ belongs to the standard numerical range $W\left(A_{11}\right)$ of $A_{11}$. So,

$$
S_{t}=\operatorname{Conv}\left\{\text { the corners of } S_{t}\right\} \subseteq W\left(A_{11}\right) .
$$

Now, using the hypothesis that $A_{12} \neq 0$, select $x \in \mathbb{C}^{k}, y \in \mathbb{C}^{n-k}$ so that $x^{*} x=1$, $x^{*} A_{12} y=\left\|A_{12} y\right\|$, and replacing, if necessary, $y$ by $\mu y$ for some $\mu>0$ we can also assume that $t=\left\|A_{11} x+A_{12} y\right\|$. Then $\left\|A_{12} y\right\| \geq t-\left\|A_{11} x\right\|$ and since $t>5\left\|A_{11}\right\|$, we have

$$
\left|x^{*} A_{11} x+x^{*} A_{12} y\right| \geq\left|x^{*} A_{12} y\right|-\left|x^{*} A_{11} x\right| \geq\left(t-\left\|A_{11} x\right\|\right)-\left\|A_{11}\right\|>\left\|A_{11}\right\| .
$$

Hence, $S_{t}$ contains $x^{*} A_{11} x+x^{*} A_{12} y$, which does not belong to $W\left(A_{11}\right)$, a contradiction with (4.3).

5. Normality and polyhedral shells: Indefinite Cases. Note that if $H$ is indefinite, $\mathcal{S}_{H}^{+}(A)$ fails to be a polyhedral set even for $H$-selfadjoint matrices. Indeed, if $H=\left[\begin{array}{cc}0 & 1 \\ 1 & 0\end{array}\right]$ and $A=\left[\begin{array}{ll}0 & 1 \\ 0 & 0\end{array}\right]$ is an $H$-selfadjoint matrix, then

$$
H A=\left[\begin{array}{ll}
0 & 0 \\
0 & 1
\end{array}\right] \quad \text { and } \quad A^{*} H A=\left[\begin{array}{ll}
0 & 0 \\
0 & 0
\end{array}\right] .
$$


Thus, letting $v=\left(v_{1}, v_{2}\right)^{T} \in \mathbb{C}^{2}$, we have that

$$
\mathcal{S}_{H}^{+}(A)=\left\{\left(\left|v_{2}\right|^{2}, 0,0\right): \operatorname{Re}\left(v_{1} \bar{v}_{2}\right)=1\right\}=\{(x, 0,0): x>0\}
$$

is an open half line, which is not a polyhedral set. This example also shows that $\mathcal{S}_{H}^{+}(A)$ need not be closed or bounded for an $H$-normal matrix $A$. Nonetheless, we have the following conjecture.

Conjecture 5.1. $\mathcal{S}_{H}^{+}(A)$ is convex and the closure of $\mathcal{S}_{H}^{+}(A)$ is a polyhedral set for every $H$-normal matrix $A$.

Conjecture 5.1 is supported by the results of the next four subsections where we explicitly compute shells of particular $H$-normal matrices. In this context, we will make use of the complete classification of $H$-normal matrices for the case when $H$ is an invertible Hermitian matrix with only one positive eigenvalue. This classification was obtained in [6].

TheOrem 5.2. Let $H$ be invertible Hermitian and let $H$ have only one positive eigenvalue. Furthermore, let $X$ be $H$-normal. Then there exists an integer $m \geq 0$ and a nonsingular matrix $P$ such that

$$
P^{-1} X P=\left[\begin{array}{cc}
X_{1} & 0 \\
0 & X_{2}
\end{array}\right], \quad P^{*} H P=\left[\begin{array}{cc}
H_{1} & 0 \\
0 & -I_{m}
\end{array}\right],
$$

where $X_{2} \in \mathbb{C}^{m \times m}$ is a diagonal matrix for $m>0$, or else $X_{2}$ and $I_{m}$ are void, and the pair $\left(X_{1}, H_{1}\right)$ is of one of the following forms:

type 1: $X_{1}=\lambda, H_{1}=1, \lambda \in \mathbb{C}$;

type 2: $X_{1}=\left[\begin{array}{cc}\lambda_{1} & 0 \\ 0 & \lambda_{2}\end{array}\right], H_{1}=\left[\begin{array}{cc}0 & 1 \\ 1 & 0\end{array}\right], \lambda_{1} \neq \lambda_{2}$;

type 3: $X_{1}=\left[\begin{array}{ll}\lambda & z \\ 0 & \lambda\end{array}\right], H_{1}=\left[\begin{array}{ll}0 & 1 \\ 1 & 0\end{array}\right], \lambda \in \mathbb{C},|z|=1$;

type 4: $X_{1}=\left[\begin{array}{ccc}\lambda & z & r \\ 0 & \lambda & z \\ 0 & 0 & \lambda\end{array}\right], H_{1}=-\left[\begin{array}{ccc}0 & 0 & 1 \\ 0 & 1 & 0 \\ 1 & 0 & 0\end{array}\right]$,

where $\lambda \in \mathbb{C}$ and either $|z|=1, r \in \mathbb{R}, 0 \leq \arg (z)<\pi$, or $z=1, r \in i \mathbb{R}$;

type 5: $X_{1}=\left[\begin{array}{cccc}\lambda & \cos (\phi) & \sin (\phi) & 0 \\ 0 & \lambda & 0 & 1 \\ 0 & 0 & \lambda & 0 \\ 0 & 0 & 0 & \lambda\end{array}\right], H_{1}=-\left[\begin{array}{llll}0 & 0 & 0 & 1 \\ 0 & 1 & 0 & 0 \\ 0 & 0 & 1 & 0 \\ 1 & 0 & 0 & 0\end{array}\right], \lambda \in \mathbb{C}$, $0<\phi \leq \frac{\pi}{2}$

Proof. This follows directly from [6, Theorem 2], by considering $X$ and $-H$.

5.1. Shells of some special $H$-normal matrices. In this subsection, we explicitly compute the shell $\mathcal{S}_{H}^{+}(X)$, where $X$ and $H$ have one of the following forms:

1) $X \in \mathbb{C}^{n \times n}$ is an upper triangular Toeplitz matrix and $H=\left[\begin{array}{ll}. & 1 \\ 1 & \end{array}\right] \in \mathbb{C}^{n \times n}$. 2) $X=\left[\begin{array}{cc}X_{1} & 0 \\ 0 & X_{2}\end{array}\right]$ and $H=\left[\begin{array}{lll} & . & 1 \\ 1 & & \end{array}\right] \in \mathbb{C}^{2 n \times 2 n}$, where $X_{1}, X_{2} \in \mathbb{C}^{n \times n}$ are 
upper triangular Toeplitz matrices.

It is easy to check that in both cases the matrix $X$ is $H$-normal. In [7] and [8] it was shown that for a large class of $H$-normal matrices a canonical form consisting of blocks of the forms 1) and 2) above can be obtained. (Those $H$-normal matrices were called block-Toeplitz $H$-normal matrices in [7]). This and the fact that blocks of the forms 1) and 2) also appear in Theorem 5.2 motivate our interest in these special $H$-normal matrices. Before computing the shells, we start with a technical result.

Proposition 5.3. Let $\alpha_{1}, \ldots, \alpha_{n} \in \mathbb{R}, \alpha_{1} \neq 0$. Then there exists a vector $v=\left(v_{1}, \ldots, v_{n}\right)^{T} \in \mathbb{C}^{n}$ such that

$$
\bar{v}_{n} v_{m}+\bar{v}_{n-1} v_{m+1}+\cdots+\bar{v}_{m} v_{n}=\alpha_{m} \quad \text { for } m=1, \ldots, n
$$

if and only if $\alpha_{n-2 k}>0$ for some $k \leq \frac{n-1}{2}$ and $a_{j}=0$ for $j=n-2 k+1, \ldots, n$.

Moreover, in this case $v$ can be chosen real.

Proof. ( $\Rightarrow$ ): Let $l \geq 0$ be such that $\alpha_{n-l} \neq 0$ and $\alpha_{n-j}=0$ for $j<l$. Then (5.1) successively implies $v_{n-j}=0$ for $j<\frac{l}{2}$. Suppose that $l=2 k+1$ is odd, then we have $v_{n}=\ldots=v_{n-k}=0$. But then (5.1) implies

$$
0=\bar{v}_{n} v_{n-2 k-1}+\cdots+\bar{v}_{n-k} v_{n-k-1}+\bar{v}_{n-k-1} v_{n-k}+\cdots+\bar{v}_{n-2 k} v_{n}=\alpha_{n-2 k-1},
$$

i.e., $\alpha_{n-l}=0$ which is a contradiction. Thus, $l$ is even, i.e., $l=2 k$ for some integer $k \geq 0$. Then we have $v_{n}=\ldots=v_{n-k+1}=0$ and $\bar{v}_{n-k} v_{n-k}=\alpha_{n-2 k}$. This implies $\alpha_{n-2 k}>0$.

$(\Leftarrow)$ : Without loss of generality, we may assume that $k=0$. Otherwise, we have $v_{n}=\ldots=v_{n-k+1}=0$ and setting $v_{1}=\ldots=v_{k}=0$ and $w_{j}=v_{k+j}$ for $j=1, \ldots, n-2 k$, equation (5.1) reduces to

$$
\bar{w}_{n-2 k} w_{m}+\bar{w}_{n-2 k-1} w_{m+1}+\cdots+\bar{w}_{m} w_{n-2 k}=\alpha_{m} \quad \text { for } m=1, \ldots, n-2 k \text {, }
$$

and we can consider the analogous problem with size $n-2 k$ instead of $n$. Then we construct the desired vector $v$ by choosing $v_{n}=\sqrt{\alpha_{n}} \in \mathbb{R}$ and computing $v_{m}$ from (5.1) successively as the solution of a linear equation of the form $2 v_{m} v_{n}=b_{m}$ for some $b_{m} \in \mathbb{R}$.

TheOREM 5.4. Let $x_{1}, \ldots, x_{n} \in \mathbb{C}, \varepsilon= \pm 1$, and

$$
H=\varepsilon\left[\begin{array}{ccc}
0 & & 1 \\
& . & \\
1 & & 0
\end{array}\right] \in \mathbb{C}^{n \times n}, \quad \text { and } \quad X=\left[\begin{array}{cccc}
x_{1} & x_{2} & \cdots & x_{n} \\
& x_{1} & \ddots & \vdots \\
& & \ddots & x_{2} \\
& & & x_{1}
\end{array}\right] \in \mathbb{C}^{n \times n}
$$

an upper triangular Toeplitz matrix. Furthermore, let

$$
\begin{aligned}
& \tilde{x}_{m}:=\bar{x}_{1} x_{m}+\bar{x}_{2} x_{m-1}+\cdots+\bar{x}_{m} x_{1}, \quad m=1, \ldots, n, \\
& \mathcal{X}_{m}:=\left(\operatorname{Re}\left(x_{m}\right), \operatorname{Im}\left(x_{m}\right), \tilde{x}_{m}\right), \quad m=1, \ldots, n, \\
& \mathcal{S}_{1}:=\operatorname{Span}\left(\mathcal{X}_{2}, \ldots, \mathcal{X}_{n-1}\right), \quad s_{1}:=\operatorname{dim}\left(\mathcal{S}_{1}\right), \\
& \mathcal{S}_{3}:=\operatorname{Span}\left(\mathcal{X}_{2}, \ldots, \mathcal{X}_{n-3}\right), \quad s_{3}:=\operatorname{dim}\left(\mathcal{S}_{3}\right), \\
& \mathcal{S}_{5}:=\operatorname{Span}\left(\mathcal{X}_{2}, \ldots, \mathcal{X}_{n-5}\right), \quad s_{5}:=\operatorname{dim}\left(\mathcal{S}_{5}\right) .
\end{aligned}
$$


TABLE 5.1

Classification of shells of H-normals of the form (5.2)

\begin{tabular}{|c|c|c|c|c|c|c|c|}
\hline$s_{1}$ & & $s_{3}$ & & $s_{5}$ & & & shell of $X$ \\
\hline 3 & & $\leq 3$ & & $\leq 3$ & & & $\mathbb{R}^{1 \times 3}$ \\
\hline 2 & $\mathcal{X}_{n} \in \mathcal{S}_{1}$ & $\leq 2$ & & $\leq 2$ & & & plane \\
\hline 2 & $\mathcal{X}_{n} \notin \mathcal{S}_{1}$ & 2 & & $\leq 2$ & & & closed half space \\
\hline 2 & $\mathcal{X}_{n} \notin \mathcal{S}_{1}$ & 1 & $\mathcal{X}_{n-2} \in \mathcal{S}_{3}$ & $\leq 1$ & & & $\begin{array}{c}\text { open half space } \\
\text { with line on boundary }\end{array}$ \\
\hline 2 & $\mathcal{X}_{n} \notin \mathcal{S}_{1}$ & 1 & $\mathcal{X}_{n-2} \notin \mathcal{S}_{3}$ & 1 & & & $\begin{array}{l}\text { open half space with } \\
\text { closed half plane o.b. }\end{array}$ \\
\hline 2 & $\mathcal{X}_{n} \notin \mathcal{S}_{1}$ & 1 & $\mathcal{X}_{n-2} \notin \mathcal{S}_{3}$ & 0 & $n=6$ & & $\begin{array}{c}\text { open half space with } \\
\text { open half plane o.b. } \\
\text { with open half line o.b. }\end{array}$ \\
\hline 2 & $\mathcal{X}_{n} \notin \mathcal{S}_{1}$ & 1 & $\mathcal{X}_{n-2} \notin \mathcal{S}_{3}$ & 0 & $n=5$ & $\varepsilon=1$ & $\begin{array}{l}\text { open half space with } \\
\text { open half plane o.b. } \\
\text { with singleton o.b. }\end{array}$ \\
\hline 2 & $\mathcal{X}_{n} \notin \mathcal{S}_{1}$ & 1 & $\mathcal{X}_{n-2} \notin \mathcal{S}_{3}$ & 0 & $n=5$ & $\varepsilon=-1$ & $\begin{array}{l}\text { open half space with } \\
\text { open half plane o.b. }\end{array}$ \\
\hline 2 & $\mathcal{X}_{n} \notin \mathcal{S}_{1}$ & 0 & & 0 & $n=4$ & & $\begin{array}{l}\text { open half space with } \\
\text { open half line o.b. }\end{array}$ \\
\hline 1 & $\mathcal{X}_{n} \in \mathcal{S}_{1}$ & $\leq 1$ & & $\leq 1$ & & & line \\
\hline 1 & $\mathcal{X}_{n} \notin \mathcal{S}_{1}$ & 1 & & $\leq 1$ & & & closed half plane \\
\hline 1 & $\mathcal{X}_{n} \notin \mathcal{S}_{1}$ & 0 & & 0 & $n=4$ & & $\begin{array}{l}\text { open half plane with } \\
\text { open half line o.b. }\end{array}$ \\
\hline 1 & $\mathcal{X}_{n} \notin \mathcal{S}_{1}$ & 0 & & 0 & $n=3$ & $\varepsilon=1$ & $\begin{array}{l}\text { open half plane with } \\
\text { singleton o.b. }\end{array}$ \\
\hline 1 & $\mathcal{X}_{n} \notin \mathcal{S}_{1}$ & 0 & & 0 & $n=3$ & $\varepsilon=-1$ & open half plane \\
\hline 0 & & 0 & & 0 & $n=2$ & & open half line \\
\hline 0 & & 0 & & 0 & $n=1$ & $\varepsilon=1$ & singleton \\
\hline 0 & & 0 & & 0 & $n=1$ & $\varepsilon=-1$ & empty set \\
\hline
\end{tabular}

Then the shell $\mathcal{S}_{H}^{+}(X)$ of $X$ has the form given by Table 5.1, where "o.b." means "on the boundary". In particular, $\mathcal{S}_{H}^{+}(X)$ is convex and its closure is a polyhedral set.

Proof. Let $v=\left(v_{1}, \ldots, v_{n}\right)^{T} \in \mathbb{C}^{n}$ be such that $\bar{v}_{n} v_{1}+\bar{v}_{n-1} v_{2}+\cdots+\bar{v}_{1} v_{n}=\varepsilon$ and set

$$
\alpha_{m}=\bar{v}_{n} v_{m}+\cdots+\bar{v}_{m} v_{n}, \quad \text { for } m=2, \ldots, n
$$

A simple computation shows that

$$
v^{*} H X v=x_{1}+\varepsilon \alpha_{2} x_{2}+\cdots+\varepsilon \alpha_{n} x_{n}, \quad v^{*} X^{*} H X v=\tilde{x}_{1}+\varepsilon \alpha_{2} \tilde{x}_{2}+\cdots+\varepsilon \alpha_{n} \tilde{x}_{n} .
$$


Thus, $S_{H}^{+}(X)$ is the set of all points

$$
\varepsilon\left(\alpha_{1} \mathcal{X}_{1}+\alpha_{2} \mathcal{X}_{2}+\cdots+\alpha_{n} \mathcal{X}_{n}\right)
$$

where $\alpha_{1}:=\varepsilon, \alpha_{2}, \ldots, \alpha_{n}$ are such that there exists $v \in \mathbb{C}^{n}$ such that (5.1) holds. Applying Proposition 5.3 and classifying the shell of $X$ with respect to the dimensions of the vector spaces $\mathcal{S}_{1}, \mathcal{S}_{3}$, and $\mathcal{S}_{5}$, and some other parameters, yields the classification of Table 5.1. $\square$

Proposition 5.5. Let $\alpha_{1}, \ldots, \alpha_{n}, \beta_{1}, \ldots, \beta_{n} \in \mathbb{R}$. Then there exists a vector $v=\left(v_{1}, \ldots, v_{2 n}\right)^{T} \in \mathbb{C}^{2 n}$ such that

$$
\bar{v}_{2 n} v_{m}+\bar{v}_{2 n-1} v_{m+1}+\cdots+\bar{v}_{n+m} v_{n}=\alpha_{m}+\beta_{m} i, \quad m=1, \ldots, n .
$$

Proof. Set $v_{2 n}=v_{2 n-1}=\ldots=v_{n+1}=1$. Then, we determine $v_{n}, \ldots, v_{1}$ successively from (5.3). $\mathrm{\square}$

THEOREM 5.6. Let $x_{1}, \ldots, x_{2 n} \in \mathbb{C}$,

$$
X_{1}=\left[\begin{array}{cccc}
x_{1} & x_{2} & \ldots & x_{n} \\
& x_{1} & \ddots & \vdots \\
& & \ddots & x_{2} \\
& & & x_{1}
\end{array}\right], \quad X_{2}=\left[\begin{array}{cccc}
x_{n+1} & x_{n+2} & \ldots & x_{2 n} \\
& x_{n+1} & \ddots & \vdots \\
& & \ddots & x_{n+2} \\
& & & x_{n+1}
\end{array}\right],
$$

upper triangular Toeplitz matrices, and

$$
X=\left[\begin{array}{cc}
X_{1} & 0 \\
0 & X_{2}
\end{array}\right], \quad H=\left[\begin{array}{ccc}
0 & & 1 \\
& . & \\
1 & & 0
\end{array}\right]_{2 n \times 2 n} .
$$

Then the shell $\mathcal{S}_{H}^{+}(X)$ of $X$ is a singleton, a line, a plane, or $\mathbb{R}^{1 \times 3}$. In particular, $\mathcal{S}_{H}^{+}(X)$ is convex and its closure is a polyhedral set.

Proof. Let $\alpha_{1}=\frac{1}{2}$ and let $\alpha_{2}, \ldots, \alpha_{n}, \beta_{1}, \ldots, \beta_{n} \in \mathbb{R}$ be arbitrary. By Proposition 5.5, there exists a vector $v=\left(v_{1}, \ldots, v_{2 n}\right)^{T} \in \mathbb{C}^{2 n}$ such that (5.3) holds. In particular, we have

$$
\bar{v}_{2 n} v_{1}+\bar{v}_{2 n-1} v_{2}+\cdots+\bar{v}_{1} v_{2 n}=\frac{1}{2}+\beta_{1} i+\frac{1}{2}-\beta_{1} i=1 .
$$

A simple computation shows

$$
\begin{aligned}
& v^{*} H X v \\
= & \left(\alpha_{1}+\beta_{1} i\right) x_{1}+\cdots+\left(\alpha_{n}+\beta_{n} i\right) x_{n}+\left(\alpha_{1}-\beta_{1} i\right) x_{n+1}+\cdots+\left(\alpha_{n}-\beta_{n} i\right) x_{2 n} \\
= & \alpha_{1}\left(x_{1}+x_{n+1}\right)+\cdots+\alpha_{n}\left(x_{n}+x_{2 n}\right)+\beta_{1} i\left(x_{1}-x_{n+1}\right)+\cdots+\beta_{n} i\left(x_{n}-x_{2 n}\right), \\
& v^{*} X^{*} H X v \\
= & \left(\alpha_{1}+\beta_{1} i\right) \tilde{x}_{1}+\cdots+\left(\alpha_{n}+\beta_{n} i\right) \tilde{x}_{n}+\left(\alpha_{1}-\beta_{1} i\right) \tilde{x}_{n+1}+\cdots+\left(\alpha_{n}-\beta_{n} i\right) \tilde{x}_{2 n} \\
= & \alpha_{1}\left(\tilde{x}_{1}+\tilde{x}_{n+1}\right)+\cdots+\alpha_{n}\left(\tilde{x}_{n}+\tilde{x}_{2 n}\right)+\beta_{1} i\left(\tilde{x}_{1}-\tilde{x}_{n+1}\right)+\cdots+\beta_{n} i\left(\tilde{x}_{n}-\tilde{x}_{2 n}\right),
\end{aligned}
$$


where

$\tilde{x}_{m}:=\bar{x}_{1} x_{n+m}+\bar{x}_{2} x_{n+m-1}+\cdots+\bar{x}_{m} x_{n+1} \quad$ and $\quad \tilde{x}_{n+m}:=\overline{\tilde{x}_{m}}, \quad$ for $m=1, \ldots, n$.

Thus, setting

$$
\begin{gathered}
\mathcal{X}_{m}=\left(\operatorname{Re}\left(x_{m}+x_{n+m}\right), \operatorname{Im}\left(x_{m}+x_{n+m}\right), \tilde{x}_{m}+\tilde{x}_{n+m}\right), \quad m=1, \ldots, n, \\
\mathcal{Y}_{m}=\left(\operatorname{Im}\left(x_{n+m}-x_{m}\right), \operatorname{Re}\left(x_{m}-x_{n+m}\right), i\left(\tilde{x}_{m}-\tilde{x}_{n+m}\right)\right), \quad m=1, \ldots, n,
\end{gathered}
$$

we obtain that

$\mathcal{S}_{H}^{+}(X)=\left\{\frac{1}{2} \mathcal{X}_{1}+\alpha_{2} \mathcal{X}_{2}+\cdots+\alpha_{n} \mathcal{X}_{n}+\beta_{1} \mathcal{Y}_{1}+\cdots+\beta_{n} \mathcal{Y}_{n}: \alpha_{2}, \ldots, \alpha_{n}, \beta_{1}, \ldots, \beta_{n} \in \mathbb{R}\right\}$.

Hence, the shell of $X$ is a singleton, a line, a plane, or $\mathbb{R}^{1 \times 3}$, depending on the dimension of the subspace spanned by $\mathcal{X}_{2}, \ldots, \mathcal{X}_{n}, \mathcal{Y}_{1}, \ldots, \mathcal{Y}_{n}$.

5.2. Shells of $2 \times 2 H$-normal matrices. The case when $H \in \mathbb{C}^{n \times n}$ is positive definite is covered in Theorem 3.1: $\mathcal{S}_{H}^{+}(A)$ is a straight line segment, whenever $A$ is $H$-normal. Now we express the coordinates of the vertices of this segment in terms of eigenvalues of the matrix $A$.

By Lemma 4.2 , if $(a, b, c)$ is a vertex of $\mathcal{S}_{H}^{+}(A)$, then

$$
\left(\frac{A+A^{*}}{2}\right)=a x, \quad\left(\frac{A-A^{*}}{2 i}\right)=b x, \quad A^{*} A x=c x
$$

for some nonzero $x \in \mathbb{R}^{2}$. It follows from the first two equalities in (5.4) that

$$
A x=(a+i b) x, \quad A^{*} x=(a-i b) x,
$$

and in particular, $a+i b$ is an eigenvalue of $A$. Since $A$ is $H$-normal, we have now from (5.5) and the third equality in (5.4)

$$
c x=A^{*} A x=A^{*}(a+i b) x=(a+i b)(a-i b) x=\left(a^{2}+b^{2}\right) x
$$

that $c=a^{2}+b^{2}$. Therefore, the shell $\mathcal{S}_{H}^{+}(A)$ is a (possibly degenerate) line segment

$$
\mathcal{S}_{H}^{+}(A)=\left\{t\left(a_{1}, b_{1}, a_{1}^{2}+b_{1}^{2}\right)+(1-t)\left(a_{2}, b_{2}, a_{2}^{2}+b_{2}^{2}\right): 0 \leq t \leq 1\right\},
$$

where $a_{1}+i b_{1}$ and $a_{2}+i b_{2}$ are eigenvalues of $A$. It can be easily shown that for a fixed $H$, the shell $\mathcal{S}_{H}^{+}(A)$ of an $H$-normal matrix $A$ is a line segment with vertices on a paraboloid and this paraboloid depends on $H$ and not on $A$.

Consider now the case when $H$ has one negative and one positive eigenvalue. Since the transformation (2.7) does not change the shell, we can use a canonical form 
for a $2 \times 2 H$-normal matrix $A$. This canonical form is a special case of the canonical form in Theorem 5.2. We are then left with the following three types of blocks.

Type 1:

$$
A=\left[\begin{array}{cc}
\lambda_{1} & 0 \\
0 & \lambda_{2}
\end{array}\right], \quad H=\left[\begin{array}{rr}
1 & 0 \\
0 & -1
\end{array}\right], \quad \lambda_{1}, \lambda_{2} \in \mathbb{C} .
$$

In this case, it follows from Proposition 2.5 (see also Theorem 3.4) that $\mathcal{S}_{H}^{+}(A)$ is a closed half line.

Type 2:

$$
A=\left[\begin{array}{cc}
\lambda_{1} & 0 \\
0 & \lambda_{2}
\end{array}\right], \quad H=\left[\begin{array}{ll}
0 & 1 \\
1 & 0
\end{array}\right], \quad \lambda_{1}, \lambda_{2} \in \mathbb{C}, \quad \lambda_{1} \neq \lambda_{2} .
$$

By Theorem 5.6, $\mathcal{S}_{H}^{+}(A)$ is a straight line.

Type 3:

$$
A=\left[\begin{array}{ll}
\lambda & z \\
0 & \lambda
\end{array}\right], \quad H=\left[\begin{array}{ll}
0 & 1 \\
1 & 0
\end{array}\right], \quad \lambda \in \mathbb{C}, \quad|z|=1
$$

By Theorem 5.4, $\mathcal{S}_{H}^{+}(A)$ is an open half line.

Finally, the case when $H$ is singular (and therefore positive semidefinite) is reduced by Theorem 2.3 to the $1 \times 1$ case. It follows that

$$
\mathcal{S}_{H}^{+}(A)=\left\{\left(\left|x_{1}\right|^{2} \operatorname{Re}(a),\left|x_{1}\right|^{2} \operatorname{Im}(a),\left|x_{1}\right|^{2}|a|^{2}\right):\left|x_{1}\right|^{2}=1\right\}=\left(\operatorname{Re}(a), \operatorname{Im}(a),|a|^{2}\right)
$$

is just a singleton. Note that the singleton always (i.e., for each $A$ ) belongs to the paraboloid $z=x^{2}+y^{2}$.

5.3. Shells of $3 \times 3 H$-normal matrices. In this subsection, we describe the shells of $3 \times 3 H$-normal matrices. Again, since transformation (2.7) does not change the shell, we can start with a canonical form for a $3 \times 3 H$-normal matrix $X$. First, let us consider the case that $H$ is nonsingular. In this case either $H$ or $-H$ has at most one positive eigenvalue. Thus, a canonical form is given in Theorem 5.2. We are then left with the discussion of the following five types of blocks.

Type 1:

$$
X=\left[\begin{array}{ccc}
\lambda_{1} & 0 & 0 \\
0 & \lambda_{2} & 0 \\
0 & 0 & \lambda_{3}
\end{array}\right], \quad H=\left[\begin{array}{ccc}
\varepsilon_{1} & 0 & 0 \\
0 & \varepsilon_{2} & 0 \\
0 & 0 & \varepsilon_{3}
\end{array}\right] \neq \pm I_{3}
$$

where $\lambda_{1}, \lambda_{2}, \lambda_{3} \in \mathbb{C}$, and $\varepsilon_{1}, \varepsilon_{2}, \varepsilon_{3} \in\{+1,-1\}$. In this case, the description follows from Theorem 3.4.

Type 2:

$$
X=\left[\begin{array}{ccc}
\lambda_{1} & 0 & 0 \\
0 & \lambda_{2} & 0 \\
0 & 0 & \lambda_{3}
\end{array}\right], \quad H=\left[\begin{array}{ccc}
\varepsilon & 0 & 0 \\
0 & 0 & 1 \\
0 & 1 & 0
\end{array}\right]
$$


where $\lambda_{1}, \lambda_{2}, \lambda_{3} \in \mathbb{C}$, and $\varepsilon \in\{+1,-1\}$. Let $v=\left(v_{1}, v_{2}, v_{3}\right)^{T} \in \mathbb{C}^{3}$ be a vector. Then we obtain that

$$
\begin{aligned}
v^{*} H v & =\varepsilon \bar{v}_{1} v_{1}+\bar{v}_{2} v_{3}+\bar{v}_{3} v_{2}, \\
v^{*} H X v & =\varepsilon \lambda_{1} \bar{v}_{1} v_{1}+\lambda_{3} \bar{v}_{2} v_{3}+\lambda_{2} \bar{v}_{3} v_{2}, \\
v^{*} X^{*} H X v & =\varepsilon \bar{\lambda}_{1} \lambda_{1} \bar{v}_{1} v_{1}+\bar{\lambda}_{2} \lambda_{3} \bar{v}_{2} v_{3}+\bar{\lambda}_{3} \lambda_{2} \bar{v}_{3} v_{2} .
\end{aligned}
$$

Setting $\alpha+i \beta:=\bar{v}_{2} v_{3}, \alpha, \beta \in \mathbb{R}$, it follows that $v^{*} H v=1$ if and only if $\varepsilon \bar{v}_{1} v_{1}=1-2 \alpha$. Hence, we have to require that $\alpha \leq \frac{1}{2}$ if $\varepsilon=1$, or $\alpha \geq \frac{1}{2}$ if $\varepsilon=-1$, respectively. Making use of

$$
\begin{aligned}
& \operatorname{Re}\left(\bar{\lambda}_{2} \lambda_{3} \bar{v}_{2} v_{3}\right) \\
= & \left(\operatorname{Re}\left(\lambda_{2}\right) \operatorname{Re}\left(\lambda_{3}\right)+\operatorname{Im}\left(\lambda_{2}\right) \operatorname{Im}\left(\lambda_{3}\right)\right) \alpha-\left(\operatorname{Re}\left(\lambda_{2}\right) \operatorname{Im}\left(\lambda_{3}\right)-\operatorname{Im}\left(\lambda_{2}\right) \operatorname{Re}\left(\lambda_{3}\right)\right) \beta,
\end{aligned}
$$

we obtain that the shell of $X$ has the form

$$
\mathcal{S}_{H}^{+}(X)=\{P+\alpha x+\beta y: \alpha, \beta \in \mathbb{R}, 2 \varepsilon \alpha \leq \varepsilon\},
$$

where

$$
\begin{gathered}
P=\left(\operatorname{Re}\left(\lambda_{1}\right), \operatorname{Im}\left(\lambda_{1}\right),\left|\lambda_{1}\right|^{2}\right), \\
x=\left(\operatorname{Re}\left(\lambda_{2}+\lambda_{3}-2 \lambda_{1}\right), \operatorname{Im}\left(-2 \lambda_{1}+\lambda_{2}+\lambda_{3}\right),\right. \\
\left.2\left(\operatorname{Re}\left(\lambda_{2}\right) \operatorname{Re}\left(\lambda_{3}\right)+\operatorname{Im}\left(\lambda_{2}\right) \operatorname{Im}\left(\lambda_{3}\right)-\left|\lambda_{1}\right|^{2}\right)\right), \\
y=\left(\operatorname{Im}\left(\lambda_{2}-\lambda_{3}\right), \operatorname{Re}\left(-\lambda_{2}+\lambda_{3}\right), 2\left(\operatorname{Im}\left(\lambda_{2}\right) \operatorname{Re}\left(\lambda_{3}\right)-\operatorname{Re}\left(\lambda_{2}\right) \operatorname{Im}\left(\lambda_{3}\right)\right)\right) .
\end{gathered}
$$

Thus, $\mathcal{S}_{H}^{+}(X)$ is a closed half plane in both cases $\varepsilon=1$ and $\varepsilon=-1$ (or a nondegenerate subset of a line, if $x$ and $y$ are linearly dependent).

Type 3:

$$
X=\left[\begin{array}{ccc}
\lambda_{1} & 0 & 0 \\
0 & \lambda_{2} & z \\
0 & 0 & \lambda_{2}
\end{array}\right], \quad H=\left[\begin{array}{ccc}
\varepsilon & 0 & 0 \\
0 & 0 & 1 \\
0 & 1 & 0
\end{array}\right],
$$

where $\lambda_{1}, \lambda_{2}, z \in \mathbb{C},|z|=1$, and $\varepsilon \in\{+1,-1\}$. Again, let $v=\left(v_{1}, v_{2}, v_{3}\right)^{T} \in \mathbb{C}^{3}$. Then we obtain that

$$
\begin{aligned}
v^{*} H v & =\varepsilon \bar{v}_{1} v_{1}+\bar{v}_{2} v_{3}+\bar{v}_{3} v_{2}, \\
v^{*} H X v & =\varepsilon \lambda_{1} \bar{v}_{1} v_{1}+\lambda_{2}\left(\bar{v}_{2} v_{3}+\bar{v}_{3} v_{2}\right)+z \bar{v}_{3} v_{3}, \\
v^{*} X^{*} H X v & =\varepsilon \bar{\lambda}_{1} \lambda_{1} \bar{v}_{1} v_{1}+\bar{\lambda}_{2} \lambda_{2}\left(\bar{v}_{2} v_{3}+\bar{v}_{3} v_{2}\right)+\left(\bar{\lambda}_{2} z+\bar{z} \lambda_{2}\right) \bar{v}_{3} v_{3} .
\end{aligned}
$$

Setting $\alpha:=\bar{v}_{1} v_{1}$ and $\beta:=\bar{v}_{3} v_{3}$, we find that $v^{*} H v=1$ if and only if $\bar{v}_{2} v_{3}+\bar{v}_{3} v_{2}=$ $1-\varepsilon \alpha$. Clearly, such vectors $v$ exist for any possible choice of $\alpha \geq 0$ and $\beta>0$. If 
$\beta=0$, i.e., $v_{3}=0$, then we have to choose $v_{1}$ such that $\varepsilon \alpha=1$, which is only possible for $\varepsilon=1$. Thus, the shell of $X$ is given by

$$
\mathcal{S}_{H}^{+}(X)=\left\{P+\alpha x+\beta y:(\alpha, \beta) \in\left\{\mathbb{R}_{0}^{+} \times \mathbb{R}^{+}\right\} \cup\{(1,0)\}\right\}
$$

for the case $\varepsilon=1$, and

$$
\mathcal{S}_{H}^{+}(X)=\left\{P-\alpha x+\beta y:(\alpha, \beta) \in \mathbb{R}_{0}^{+} \times \mathbb{R}^{+}\right\}
$$

for the case $\varepsilon=-1$, where $P=\left(\operatorname{Re}\left(\lambda_{2}\right), \operatorname{Im}\left(\lambda_{2}\right),\left|\lambda_{2}\right|^{2}\right), x=\left(\operatorname{Re}\left(\lambda_{1}-\lambda_{2}\right), \operatorname{Im}\left(\lambda_{1}-\right.\right.$ $\left.\left.\lambda_{2}\right),\left|\lambda_{1}\right|^{2}-\left|\lambda_{2}\right|^{2}\right), y=\left(\operatorname{Re}(z), \operatorname{Im}(z), \bar{\lambda}_{2} z+\bar{z} \lambda_{2}\right)$. We sketch $\mathcal{S}_{H}^{+}(X)$ in Figure 5.1 as subsets of the plane $E=\{P+\alpha x+\beta y: \alpha, \beta \in \mathbb{R}\}$, for the cases a) $\varepsilon=1$ and b) $\varepsilon=-1$, assuming that $x$ and $y$ are linearly independent. Note that in case a) only one point on the half line $\alpha x$, is an element of $\mathcal{S}_{H}^{+}(X)$, while in case b) the whole half line $-\alpha x$ is excluded from $\mathcal{S}_{H}^{+}(X)$.

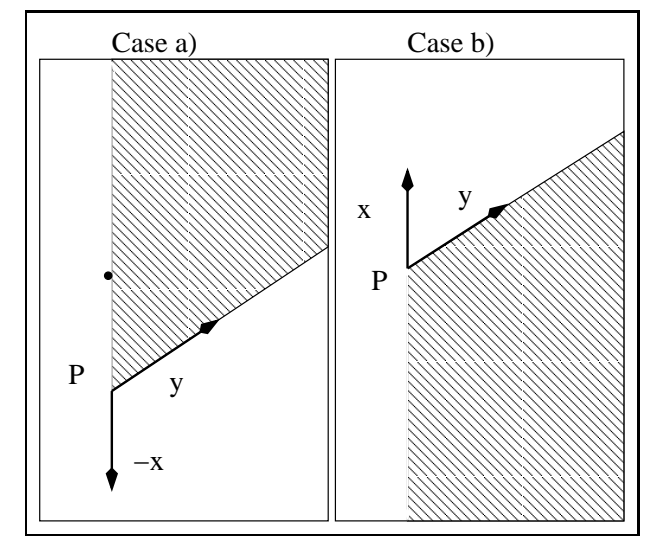

FIG. 5.1. Shells of $3 \times 3 H$-normal matrices of type 3

Type 4:

$$
X=\left[\begin{array}{lll}
\lambda & z & r \\
0 & \lambda & z \\
0 & 0 & \lambda
\end{array}\right], \quad H=\varepsilon\left[\begin{array}{lll}
0 & 0 & 1 \\
0 & 1 & 0 \\
1 & 0 & 0
\end{array}\right], \quad \varepsilon= \pm 1
$$

where $\lambda, z \in \mathbb{C},|z|=1,0 \leq \arg z<\pi$, and $r \in \mathbb{R}$. By Theorem 5.4, $\mathcal{S}_{H}^{+}(X)$ is the union of an open half plane and a singleton on its boundary. We describe $\mathcal{S}_{H}^{+}(X)$ in more detail. Let $v=\left(v_{1}, v_{2}, v_{3}\right)^{T} \in \mathbb{C}^{3}$. Then

$$
\begin{aligned}
v^{*} H v & =\bar{v}_{3} v_{1}+\bar{v}_{2} v_{2}+\bar{v}_{1} v_{3}, \\
v^{*} H X v & =\lambda\left(\bar{v}_{3} v_{1}+\bar{v}_{2} v_{2}+\bar{v}_{1} v_{3}\right)+z\left(\bar{v}_{3} v_{2}+\bar{v}_{2} v_{3}\right)+r \bar{v}_{3} v_{3}, \\
v^{*} X^{*} H X v & =\bar{\lambda} \lambda\left(\bar{v}_{3} v_{1}+\bar{v}_{2} v_{2}+\bar{v}_{1} v_{3}\right)+(\lambda \bar{z}+\bar{\lambda} z)\left(\bar{v}_{3} v_{2}+\bar{v}_{2} v_{3}\right)+(r \bar{\lambda}+r \lambda+1) \bar{v}_{3} v_{3} .
\end{aligned}
$$

Setting $\beta:=\bar{v}_{3} v_{3}$ and $\alpha:=\bar{v}_{3} v_{2}+\bar{v}_{2} v_{3}$, we find that vectors $v$ satisfying $v^{*} H v=1$ can be found for all possible values of $\alpha$ if $\beta>0$. Only if $\beta=0$, i.e., $v_{3}=0$, then we 
have to require $\alpha=0$ and $\left|v_{2}\right|=1$. Thus, the shell of $X$ has the form

$$
\mathcal{S}_{H}^{+}(X)=\left\{P+\alpha x+\beta y:(\alpha, \beta) \in \mathbb{R} \times \mathbb{R}^{+} \cup\{(0,0)\}\right\},
$$

where

$$
P=(\operatorname{Re}(\lambda),-\operatorname{Im}(\lambda), \bar{\lambda} \lambda), x=(\operatorname{Re}(z),-\operatorname{Im}(z), \lambda \bar{z}+\bar{\lambda} z), y=(r, 0, r \bar{\lambda}+r \lambda+1) .
$$

(Note that $x$ and $y$ are linearly independent for all possible values of $r \in \mathbb{R}$ and $z \in \mathbb{C}$ with $|z|=1$.)

Type 5:

$$
X=\left[\begin{array}{ccc}
\lambda & 1 & i r \\
0 & \lambda & 1 \\
0 & 0 & \lambda
\end{array}\right], \quad H=\varepsilon\left[\begin{array}{lll}
0 & 0 & 1 \\
0 & 1 & 0 \\
1 & 0 & 0
\end{array}\right], \quad \varepsilon= \pm 1,
$$

where $\lambda \in \mathbb{C}$ and $r \in \mathbb{R}$. By Theorem 5.4, $\mathcal{S}_{H}^{+}(X)$ is the union of an open half plane and the singleton on its boundary.

Now we turn to the case when $H$ is singular. By Lemma 2.2, we need only to consider three different cases:
1) $H=\left[\begin{array}{lll}1 & 0 & 0 \\ 0 & 0 & 0 \\ 0 & 0 & 0\end{array}\right]$,
2) $H=\left[\begin{array}{lll}1 & 0 & 0 \\ 0 & 1 & 0 \\ 0 & 0 & 0\end{array}\right]$
3) $H=\left[\begin{array}{rrr}1 & 0 & 0 \\ 0 & -1 & 0 \\ 0 & 0 & 0\end{array}\right]$.

The first two cases are reduced to shells of $1 \times 1$ and $2 \times 2$ matrices by Theorem 2.3. Thus, we consider the third case only.

Case 3: Let $H_{1}=\left[\begin{array}{rr}1 & 0 \\ 0 & -1\end{array}\right]$. It follows from the definition that $A \in \mathbb{C}^{3 \times 3}$ is $H$-normal if and only if it is of the form

$$
A=\left[\begin{array}{c|c}
A_{1} & c \\
\hline * & * \\
\hline *
\end{array}\right]
$$

where $A_{1} \in \mathbb{C}^{2 \times 2}$ is $H_{1}$-normal, and moreover,

$$
|c|=|d| \quad \text { and } \quad A_{1}^{*} H_{1}\left[\begin{array}{c}
c \\
d
\end{array}\right]=0 .
$$

If $c=d=0$, then

$$
H A=\left[\begin{array}{cc}
H_{1} A_{1} & 0 \\
0 & 0
\end{array}\right], \quad A^{*} H A=\left[\begin{array}{cc}
A_{1}^{*} H_{1} A_{1} & 0 \\
0 & 0
\end{array}\right]
$$

and therefore

$$
\mathcal{S}_{H}^{+}(A)=\mathcal{S}_{H_{1}}^{+}\left(A_{1}\right)
$$


Since $A_{1}$ is a $2 \times 2 H_{1}$-normal matrix, it follows from the analysis in Section 5 that $\mathcal{S}_{H}^{+}(A)$ is a line or a half line (open or closed). In particular we have these kinds of shells if $\operatorname{det} A_{1} \neq 0$, since then we have by the second relation in (5.7) that $c=d=0$.

Now let $\operatorname{det} A_{1}=0$ and $|c|=|d| \neq 0$. Setting $\delta=\bar{d} / \bar{c}$ (note that by the first relation in $(5.7),|\delta|=1$ ) we conclude from the second relation in (5.7) that

$$
A_{1}=\left[\begin{array}{cc}
\delta \alpha & \delta \beta \\
\alpha & \beta
\end{array}\right]
$$

for some $\alpha, \beta \in \mathbb{C}$. Therefore,

$$
A_{1}^{*} H_{1} A_{1}=0 .
$$

By (5.6), (5.7), and (5.9),

$$
H A=\left[\begin{array}{c|c}
H_{1} A_{1} & c \\
& -d \\
\hline * & *
\end{array}\right], \quad A^{*} H A=\left[\begin{array}{cc}
A_{1}^{*} H_{1} A_{1} & 0 \\
0 & 0
\end{array}\right]=0,
$$

and therefore, upon setting $x=\left(x_{1}, x_{2}, x_{3}\right)^{T} \in \mathbb{C}^{3}$,

$$
\begin{aligned}
& \mathcal{S}_{H}^{+}(A) \\
& =\left\{\left(\left[\begin{array}{ll}
\overline{x_{1}} & -\overline{x_{2}}
\end{array}\right] A_{1}\left[\begin{array}{l}
x_{1} \\
x_{2}
\end{array}\right]+c\left[\overline{x_{1}} \quad \overline{x_{2}}\right]\left[\begin{array}{r}
\frac{1}{\delta}
\end{array}\right] x_{3}, 0\right):\left|x_{1}\right|^{2}-\left|x_{2}\right|^{2}=1\right\} .
\end{aligned}
$$

Since $x_{1}-x_{2} \delta \neq 0$ for some choice of $x_{1}$ and $x_{2}$ subject to $\left|x_{1}\right|^{2}-\left|x_{2}\right|^{2}=1$ it follows that $\mathcal{S}_{H}^{+}(A)$ is the coordinate $x y$-plane.

In particular, we obtain the following conclusion from the above discussion.

THEOREM 5.7. Let $A$ be an $n \times n H$-normal matrix, where $n \leq 3$. Then the shell $\mathcal{S}_{H}^{+}(A)$ is a subset of a line if $n=2$, and a subset of a plane if $n=3$. Moreover, $\mathcal{S}_{H}^{+}(A)$ is convex, and its closure is a polyhedral set.

Thus, Conjecture 5.1 holds true for $H$-normal matrices of sizes less than 4 .

5.4. The case $H$ has only one positive eigenvalue. In this subsection, we will prove Conjecture 5.1 for the case that $H$ is an invertible Hermitian matrix with only one positive eigenvalue. First, let us focus on the blocks of type 5 of Theorem 5.2. These matrices have been extensively used in [15] as a counter example for many statements on $H$-normal matrices that are true for the case that $H$ is positive definite. However, we next show that Conjecture 5.1 still holds true.

Proposition 5.8. Let $\lambda \in \mathbb{C}, 0<\phi \leq \frac{\pi}{2}$, and

$$
X=\left[\begin{array}{cccc}
\lambda & \cos (\phi) & \sin (\phi) & 0 \\
0 & \lambda & 0 & 1 \\
0 & 0 & \lambda & 0 \\
0 & 0 & 0 & \lambda
\end{array}\right], \quad H=\varepsilon\left[\begin{array}{cccc}
0 & 0 & 0 & 1 \\
0 & 1 & 0 & 0 \\
0 & 0 & 1 & 0 \\
1 & 0 & 0 & 0
\end{array}\right], \quad \varepsilon= \pm 1
$$

Then the shell $\mathcal{S}_{H}^{+}(X)$ of $X$ is convex and its closure is a polyhedral set. 
Proof. Using Proposition 2.1(b), we assume $\lambda=0$. Let $v=\left(v_{1}, v_{2}, v_{3}, v_{4}\right)^{T} \in \mathbb{C}^{4}$ such that $v^{*} H v=1$ and let be $\alpha, \beta, \gamma, \delta, \eta \in \mathbb{R}, \eta \geq 0$ such that

$$
\bar{v}_{4} v_{2}=\alpha+i \beta, \quad \bar{v}_{4} v_{3}=\gamma+i \delta, \quad \bar{v}_{4} v_{4}=\eta .
$$

Note that, on the other hand, for any choice of $\alpha, \beta, \gamma, \delta, \eta \in \mathbb{R}, \eta>0$, there exists a vector $v \in \mathbb{C}^{4}$ such that (5.1) and $\bar{v}_{4} v_{1}+\bar{v}_{2} v_{2}+\bar{v}_{3} v_{3}+\bar{v}_{1} v_{4}=\varepsilon$ hold, for example, take

$$
v_{4}=\sqrt{\eta}, \quad v_{2}=\frac{\alpha+i \beta}{v_{4}}, \quad v_{3}=\frac{\gamma+i \delta}{v_{4}}, \quad v_{1}=\frac{\varepsilon-\left|v_{2}\right|^{2}-\left|v_{3}\right|^{2}}{2 v_{4}}
$$

In the case $\eta=0$, we must have $v_{4}=0$ and therefore, such a $v \in \mathbb{C}^{4}$ exists if and only if $\alpha=\beta=\gamma=\delta=0$. A simple computation shows that

$$
\begin{gathered}
v^{*} H X v=\bar{v}_{4} v_{2} \cos (\phi)+\bar{v}_{4} v_{3} \sin (\phi)+\bar{v}_{2} v_{4}=(\alpha+i \beta) \cos (\phi)+(\gamma+i \delta) \sin (\phi)+\alpha-i \beta, \\
v^{*} X^{*} H X v=\bar{v}_{4} v_{4}=\eta .
\end{gathered}
$$

Thus, setting $x_{1}=(\cos (\phi)+1,0,0), x_{2}=(0, \cos (\phi)-1,0), x_{3}=(\sin (\phi), 0,0)$, $x_{4}=(0, \sin (\phi), 0)$, and $x_{5}=(0,0,1)$, and noting that $x_{1}$ and $x_{3}\left(x_{2}\right.$ and $x_{4}$, respectively) are linearly dependent, we obtain that

$$
\mathcal{S}_{H}^{+}(X)=\left\{\alpha_{1} x_{1}+\alpha_{2} x_{2}+\alpha_{3} x_{5}:\left(\alpha_{1}, \alpha_{2}, \alpha_{3}\right) \in\left(\mathbb{R} \times \mathbb{R} \times \mathbb{R}^{+}\right) \cup\{(0,0,0)\}\right\} .
$$

Clearly this set is convex and its closure is polyhedral. $\square$

For the proof of the main result in this subsection, we will need the following observation.

Lemma 5.9. Let $H \in \mathbb{C}^{n \times n}$ be Hermitian, $X \in \mathbb{C}^{n \times n}$, and $v \in \mathbb{C}^{n}$. If $v^{*} H v=$ $\alpha>0$, then $\tilde{v}:=\frac{1}{\sqrt{\alpha}} v$ satisfies $\tilde{v}^{*} H \tilde{v}=1$ and

$$
v^{*} H X v=\alpha \tilde{v}^{*} H X \tilde{v}, \quad v^{*} X^{*} H X v=\alpha \tilde{v}^{*} X^{*} H X \tilde{v} .
$$

Consequently, for $\alpha>0$, we have

$$
\left\{\left(v^{*} H X v, v^{*} X^{*} H X v\right): v \in \mathbb{C}^{n}, v^{*} H v=\alpha\right\}=\alpha \mathcal{S}_{H}^{+}(X) .
$$

THEOREM 5.10. Let $H$ be invertible Hermitian and let $H$ have only one positive eigenvalue. Then the shell $\mathcal{S}_{H}^{+}(X)$ of an $H$-normal matrix $X$ is convex and its closure is a polyhedral set.

Proof. Without loss of generality, we may assume that $X$ and $H$ are in the form of Theorem 5.2. If $X$ and $H$ are one of the blocks of type 1-5, then the result follows from our discussion in the previous subsections or from Proposition 5.8. Hence, let us assume that $X=\operatorname{diag}\left(X_{1}, X_{2}\right)$ and $H=\operatorname{diag}\left(H_{1},-I_{m}\right)$ are such that $X_{2} \in$ $\mathbb{C}^{m \times m}, m>0$, is diagonal and such that the pair $\left(X_{1}, H_{1}\right)$ is of one of the forms of 
type $1-5$ of Theorem 5.2. Let $v=\left(v_{1}, v_{2}\right)^{T}$ be partitioned conformably with $X$ and $H$. Then

$$
v^{*} H v=1 \quad \Longleftrightarrow \quad v_{1}^{*} H_{1} v_{1}=1+v_{2}^{*} v_{2} .
$$

Thus, we obtain that the shell $\mathcal{S}_{H}^{+}(X)$ of $X$ is the set

$$
\begin{aligned}
& \mathcal{S}_{H}^{+}(X) \\
= & \left\{\left(v^{*} H X v, v^{*} X^{*} H X v\right): v^{*} H v=1\right\} \\
= & \left\{\left(v_{1}^{*} H_{1} X_{1} v_{1}, v_{1}^{*} X_{1}^{*} H_{1} X_{1} v_{1}\right)-\left(v_{2}^{*} X_{2} v_{2}, v_{2}^{*} X_{2}^{*} X_{2} v_{2}\right): v^{*} H v=1, v=\left(\begin{array}{c}
v_{1} \\
v_{2}
\end{array}\right)\right\} \\
= & \left.\bigcup_{\alpha \geq 0}\left((1+\alpha) \mathcal{S}_{H_{1}}^{+}\left(X_{1}\right)-\alpha \mathcal{S}_{I}^{+}\left(X_{2}\right)\right) \quad \text { (by Lemma } 5.9 \text { with } \alpha=v_{2}^{*} v_{2}\right) .
\end{aligned}
$$

Using the results in the previous subsections and Proposition 5.8, we obtain that $\mathcal{S}_{H_{1}}^{+}\left(X_{1}\right)$ consists of all the points of the form

$$
\mathcal{X}_{0}+\alpha_{1} \mathcal{X}_{1}+\alpha_{2} \mathcal{X}_{2}+\alpha_{3} \mathcal{X}_{3}
$$

where $\mathcal{X}_{i} \in \mathbb{R}^{1 \times 3}(i=0,1,2,3)$ are fixed, and $\left(\alpha_{1}, \alpha_{2}, \alpha_{3}\right) \in \mathcal{M}$. Here, $\mathcal{M}$ stands for one of the sets $\{0\} \times\{0\} \times\{0\}, \mathbb{R} \times\{0\} \times\{0\}, \mathbb{R}^{+} \times\{0\} \times\{0\},\left(\mathbb{R} \times \mathbb{R}^{+} \times\{0\}\right) \cup\{0,0,0\}$, or $\left(\mathbb{R} \times \mathbb{R} \times \mathbb{R}^{+}\right) \cup\{0,0,0\}$. Moreover, we know that $\mathcal{S}_{I}^{+}\left(X_{2}\right)$ is a polyhedron, i.e., it has the form

$$
\mathcal{S}_{I}^{+}\left(X_{2}\right)=\left\{\beta_{1} \mathcal{Y}_{1}+\ldots+\beta_{l} \mathcal{Y}_{l}: \beta_{i} \geq 0, \sum_{i=1}^{l} \beta_{i}=1\right\} .
$$

Hence, $\mathcal{S}_{H}^{+}(X)$ consists of all the points of the form

$$
\begin{aligned}
& (1+\alpha)\left(\mathcal{X}_{0}+\alpha_{1} \mathcal{X}_{1}+\alpha_{2} \mathcal{X}_{2}+\alpha_{3} \mathcal{X}_{3}\right)-\alpha\left(\beta_{1} \mathcal{Y}_{1}+\ldots+\beta_{l} \mathcal{Y}_{l}\right) \\
= & \mathcal{X}_{0}+\alpha \alpha_{1} \mathcal{X}_{1}+\alpha \alpha_{2} \mathcal{X}_{2}+\alpha \alpha_{3} \mathcal{X}_{3}+\alpha \beta_{1} \mathcal{Z}_{1}+\ldots+\alpha \beta_{l} \mathcal{Z}_{l},
\end{aligned}
$$

where $\alpha \geq 0,\left(\alpha_{1}, \alpha_{2}, \alpha_{3}\right) \in \mathcal{M}, \mathcal{Z}_{i}=\mathcal{X}_{0}-\mathcal{Y}_{i}, \beta_{i} \geq 0, i=1, \ldots, l, \sum_{i=1}^{l} \beta_{i}=1$. Consequently,

$\mathcal{S}_{H}^{+}(X)=\left\{\mathcal{X}_{0}+\tilde{\alpha}_{1} \mathcal{X}_{1}+\tilde{\alpha}_{2} \mathcal{X}_{2}+\tilde{\alpha}_{3} \mathcal{X}_{3}+\tilde{\beta}_{1} \mathcal{Z}_{1}+\ldots+\tilde{\beta}_{l} \mathcal{Z}_{l}:\left(\tilde{\alpha}_{1}, \tilde{\alpha}_{2}, \tilde{\alpha}_{3}\right) \in \mathcal{M}, \tilde{\beta}_{i} \geq 0\right\}$.

Clearly, this set is convex. The closure of $\mathcal{S}_{H}^{+}(X)$ is finitely generated (in the terminology of [16]) and hence polyhedral ([16, Theorem 19.1]).

6. Infinite dimensional case. The concept of the shell makes sense also for operators in infinite dimensional Hilbert spaces. Thus, let $\mathcal{H}$ be an infinite dimensional complex Hilbert space with the inner product $\langle x, y\rangle$, and let $H$ be a fixed bounded selfadjoint operator on $\mathcal{H}$ which is not negative semidefinite. For a (linear bounded) operator $A$ on $\mathcal{H}$ define

$$
\mathcal{S}_{H}^{+}(A)=\left\{\left([A v, v]_{H},[A v, A v]_{H}\right) \subseteq \mathbb{C} \times \mathbb{R}: v \in \mathcal{H},[v, v]_{H}=1\right\},
$$


where $[x, y]_{H}=\langle H x, y\rangle, x, y \in \mathcal{H}$. Many results of Sections 2 and 3 admit straightforward generalization to the infinite dimensional case, or parallel results for this case can be developed. We note that $H$-normal operators are defined (via (2.5)) only if $H$ has a Moore-Penrose inverse, and therefore this hypothesis is implicitly assumed each time a $H$-normal operator appears. As it is well-known, $H$ has a Moore-Penrose inverse if and only if the range of $H$ is a closed subspace. Thus, the results of Section 2 (except Proposition 2.5) go over to the infinite dimensional case essentially without changes.

Next, consider the geometric properties of shells. Theorem 3.3 is obviously valid also in the infinite dimensional case. To prove Theorem 3.5(a) in this case, assume first that $\mathcal{S}_{H}^{+}(A)$ is a singleton. Fix $v \in \mathcal{H}$ such that $[v, v]_{H}=1$. Then for every $u \in \mathcal{H}$ and for every $\alpha \in \mathbb{C}$ sufficiently close to zero, $[v+\alpha u, v+\alpha u]_{H}>0$. Scaling $v+\alpha u$ appropriately, and using the property that $\mathcal{S}_{H}^{+}(A)$ is a singleton, we obtain

$$
\langle H A(v+\alpha u), v+\alpha u\rangle=\langle H(v+\alpha u), v+\alpha u\rangle\langle H A v, v\rangle .
$$

Letting $\alpha$ be real, it follows that $\langle H A u, u\rangle=\langle H u, u\rangle\langle H A v, v\rangle$ by equating coefficients of $\alpha^{2}$. Since $u \in \mathcal{H}$ is arbitrary, we must have $H A=c H, c \in \mathbb{C}$, as required.

For Theorem 3.5(b), first observe that if the operators $H, H A+A^{*} H, i(H A-$ $\left.A^{*} H\right), A^{*} H A$ span a two dimensional real subspace, then $\mathcal{S}_{H}^{+}(A)$ is easily seen to be a subspace of a line. Conversely, assume $\mathcal{S}_{H}^{+}(A) \subseteq\{r+t q: t \in \mathbb{R}\}$ for some vectors $r, q \in \mathbb{R}^{1 \times 3}$. Applying transformations of Proposition 2.1, we may without loss of generality assume that two out of the three components of $q$ are zeros. Say for instance, the first and the third components of $q$ are zero (in other cases, the proof is analogous). Then with $u, v$, and $\alpha$ as in the preceding paragraph, we have

$$
\begin{gathered}
\left\langle\left(H A+A^{*} H\right)(v+\alpha u), v+\alpha u\right\rangle=\langle H(v+\alpha u), v+\alpha u\rangle\left\langle\left(H A+A^{*} H\right) v, v\right\rangle, \\
\langle H A(v+\alpha u), A(v+\alpha u)\rangle=\langle H(v+\alpha u), v+\alpha u\rangle\langle H A v, A v\rangle .
\end{gathered}
$$

Again, equating the coefficients of $\alpha^{2}$ in both sides of each of these equalities, we obtain that $H A+A^{*} H$ and $A^{*} H A$ are scalar multiples of $H$, concluding the proof.

Finally, consider boundedness. Recall the definition (introduced in [14]) of the numerical range with respect to the indefinite inner product induced by $H$ :

$$
W_{H}^{+}(A)=\left\{[A v, v]_{H}: v \in \mathcal{H},[v, v]_{H}=1\right\} .
$$

THEOREM 6.1. Let $H$ be a selfadjoint operator on $\mathcal{H}$, not negative semidefinite. Then the following statements are equivalent for an operator $A$ on $\mathcal{H}$ :

(1) The numerical range $W_{H}^{+}(A)$ is bounded.

(2) The shell $\mathcal{S}_{H}^{+}(A)$ is bounded.

(3) EITHER $H$ is indefinite and $H A=\alpha H$ for some $\alpha \in \mathbb{C}$, OR the properties (i) and (ii) below are satisfied: ( $i) H$ is positive semidefinite; (ii) the linear set Range $(\sqrt{H})$ is $A^{*}$-invariant, where $\sqrt{H}$ is the positive semidefinite square root of $H$.

Proof. (1) $\Leftrightarrow(3)$ is proved in [11, Theorem 2.3]. (2) $\Rightarrow(1)$ is obvious. Finally, (3) $\Rightarrow$ (2) follows easily, because by Douglas's lemma [5], (i) and (ii) imply that there exists $\lambda \geq 0$ such that $\lambda H-A^{*} H A$ is positive semidefinite. 
7. A Maple program. Here we include a Maple program which displays a portion of the shell $S_{H}^{+}(A)$ that helped us to formulate and check conjectures at the early stage of our project.

The procedures are limited to parameterization of a vector based on variables $r$ and $\alpha$. In plotshell2 one can specify $v$, however, the ranges of $r$ and $\alpha$ are fixed to $r=-10 \cdot 10$ and $\alpha=-2 \pi \cdot 2 \pi$, but $X$ (and correspondingly $v$ ) may have arbitrary dimensions. In plotshell the vector $v$ is fixed to $v=\left[\begin{array}{c}r e^{i \alpha} \\ i\end{array}\right]$, but one can change the ranges of $r$ and $\alpha$.

$>$ restart;with(plots):with(linalg):

Warning, new definition for norm

Warning, new definition for trace

$>$ \# This procedure, takes a $\mathrm{H}$ for the hermitian

$>$ \# matrix that defines the inner

$>$ \# product, an $\mathrm{X}$ for the matrix, and a $\mathrm{V}$ given

$>$ \# by the user ' $a$ ' represents the magnitude

$>$ \# of $\mathrm{v}$ since we only want $[\mathrm{v}, \mathrm{v}]=1 \mathrm{~b}$ is the

$>$ \# numerical range not divided by the norm of $\mathrm{v}$

$>\# \mathrm{x} 1, \mathrm{x} 2$ are the real and imaginary components of the

$>$ \# numerical range $\mathrm{x} 3$ is the second component $[\mathrm{Xv}, \mathrm{Xv}]$

$>$ \# This plots $\mathrm{x} 1, \mathrm{x} 2, \mathrm{x} 3$ parameterized by a

$>$ \# vector that depends on a phase alpha and a magnitude $r$

$>$ plotshell2 := proc $(\mathrm{H}, \mathrm{X}, \mathrm{V})$

local a, b, x1, x2, x3;

a:=simplify (multiply (htranspose (V) , H,V));

$\mathrm{b}:=\operatorname{simplify}(\operatorname{multiply}($ htranspose $(\mathrm{V}), \mathrm{H}, \mathrm{X}, \mathrm{V}))$;

$\mathrm{x} 1:=\operatorname{simplify}(\operatorname{Re}(\mathrm{b}[1,1]) / \mathrm{a}[1,1])$;

$\mathrm{x} 2:=\operatorname{simplify}(\operatorname{Im}(\mathrm{b}[1,1]) / \mathrm{a}[1,1])$;

$>$ x3:=simplify (multiply (htranspose (V), htranspose (X), H,X,V)) $[1,1]$ $/ \mathrm{a}[1,1]$

$>\operatorname{plot} 3 d([\mathrm{x} 1, \mathrm{x} 2, \mathrm{x} 3]$, alpha $=-2 * \mathrm{Pi} .2 * \mathrm{Pi}, \mathrm{r}=-10 \ldots 10)$; end;

$>$

$>$ \# This is a similar procedure only the $\mathrm{v}$ is fixed,

$>$ \# but you can change the parameterization values or $r$

$>$ \# and alpha by calling it with an rlow rhigh

$>$ \# and alphalow and alphahigh

$>$

$>$ plotshell := proc(H, X, rlow,rhigh,alphalow,alphahigh)

$>$ local a, b, x1, x2, x3,V;

assume ( $r$, real, alpha, real):

$\mathrm{V}:=\operatorname{matrix}([[\mathrm{r} *(\cos (\mathrm{alpha})+\mathrm{I} * \sin (\mathrm{alpha}))],[1]])$;

a:=simplify (multiply (htranspose (V), H,V));

$\mathrm{b}:=\operatorname{simplify}($ multiply (htranspose (V), H,X,V)) ;

$>\mathrm{x} 1:=\operatorname{simplify}(\operatorname{Re}(\mathrm{b}[1,1]) / \mathrm{a}[1,1])$; 
$\mathrm{x} 2:=\operatorname{simplify}(\operatorname{Im}(\mathrm{b}[1,1]) / \mathrm{a}[1,1])$;

$>$ x3:=simplify (multiply (htranspose (V), htranspose (X), H,X,V)) $[1,1]$

$/ \mathrm{a}[1,1]$;

$>\operatorname{plot} 3 d([x 1, x 2, x 3]$, alpha $=$ alphalow $\ldots$ alphahigh,r= rlow. rhigh $)$ end;

\section{REFERENCES}

[1] P. Binding and C. K. Li. Joint ranges of Hermitian matrices and simultaneous diagonalization. Linear Algebra Appl., 151:157-168, 1991.

[2] M.-T. Chien and H. Nakazato. Davis-Wielandt shell and $q$-numerical range. Linear Algebra Appl., 340:15-31, 2002.

[3] C. Davis. The shell of a Hilbert-space operator. Acta Sci. Math.(Szeged), 29:69-86, 1968.

[4] C. Davis. The shell of a Hilbert-space operator. II. Acta Sci. Math. (Szeged), 31:301-318, 1970.

[5] R. G. Douglas. On majorization, factorization, and range inclusion of operators in Hilbert space. Proc. Amer. Math. Soc., 17:413-415, 1966.

[6] I. Gohberg and B. Reichstein. On classification of normal matrices in an indefinite scalar product. Integral Equations Operator Theory, 13:364-394, 1990.

[7] I. Gohberg and B. Reichstein. On H-unitary and block-Toeplitz H-normal operators. Linear and Multilinear Algebra, 30:17-48, 1991.

[8] I. Gohberg and B. Reichstein. Classification of block-Toeplitz H-normal operators. Linear and Multilinear Algebra, 34:213-245, 1993.

[9] R. A. Horn and C. R. Johnson. Topics in Matrix Analysis. Cambridge University Press, Cambridge, 1991.

[10] C. K. Li and H. Nakazato. Some results on the $q$-numerical range. Linear and Multilinear Algebra, 43: 385-409, 1998.

[11] C. K. Li and L. Rodman. Remarks on numerical ranges of operators in spaces with an indefinite metric. Proc. Amer. Math. Soc., 126:973-982, 1998.

[12] C. K. Li and L. Rodman. Shapes and computer generation of numerical ranges of Krein space operators. Electronic Linear Algebra, 3:31-47, 1998.

[13] C. K. Li and L. Rodman. H-joint numerical ranges, to appear in Bull. of Australian Math. Soc. (Preprint available at http://www.resnet.wm.edu/ cklixx/jhw.pdf).

[14] C. K. Li, N. K. Tsing, and F. Uhlig. Numerical ranges of an operator on an indefinite inner product space. Electronic Linear Algebra, 1:1-17, 1996.

[15] C. Mehl and L. Rodman. Classes of matrices in indefinite inner products. Linear Algebra Appl., 336:71-98, 2001.

[16] R. T. Rockafellar. Convex Analysis. Princeton University Press, Princeton, NJ, 1970. 